TRANSACTIONS OF THE

AMERICAN MATHEMATICAL SOCIETY

Volume 358, Number 8, Pages 3591-3622

S 0002-9947(06)03812-8

Article electronically published on January 24, 2006

\title{
FROBENIUS MORPHISMS AND REPRESENTATIONS OF ALGEBRAS
}

\author{
BANGMING DENG AND JIE DU
}

\begin{abstract}
By introducing Frobenius morphisms $F$ on algebras $A$ and their modules over the algebraic closure $\overline{\mathbb{F}}_{q}$ of the finite field $\mathbb{F}_{q}$ of $q$ elements, we establish a relation between the representation theory of $A$ over $\overline{\mathbb{F}}_{q}$ and that of the $F$-fixed point algebra $A^{F}$ over $\mathbb{F}_{q}$. More precisely, we prove that the category mod- $A^{F}$ of finite-dimensional $A^{F}$-modules is equivalent to the subcategory of finite-dimensional $F$-stable $A$-modules, and, when $A$ is finite dimensional, we establish a bijection between the isoclasses of indecomposable $A^{F}$-modules and the $F$-orbits of the isoclasses of indecomposable $A$-modules. Applying the theory to representations of quivers with automorphisms, we show that representations of a modulated quiver (or a species) over $\mathbb{F}_{q}$ can be interpreted as $F$-stable representations of the corresponding quiver over $\overline{\mathbb{F}}_{q}$. We further prove that every finite-dimensional hereditary algebra over $\mathbb{F}_{q}$ is Morita equivalent to some $A^{F}$, where $A$ is the path algebra of a quiver $Q$ over $\overline{\mathbb{F}}_{q}$ and $F$ is induced from a certain automorphism of $Q$. A close relation between the Auslander-Reiten theories for $A$ and $A^{F}$ is established. In particular, we prove that the Auslander-Reiten (modulated) quiver of $A^{F}$ is obtained by "folding" the Auslander-Reiten quiver of $A$. Finally, by taking Frobenius fixed points, we are able to count the number of indecomposable representations of a modulated quiver over $\mathbb{F}_{q}$ with a given dimension vector and to generalize Kac's theorem for all modulated quivers and their associated Kac-Moody algebras defined by symmetrizable generalized Cartan matrices.
\end{abstract}

\section{Introduction}

In his work [14, Gabriel introduced the idea of quiver representations and discovered a remarkable connection between the indecomposable representations of (simply-laced) Dynkin quivers and the positive roots of the corresponding finitedimensional simple Lie algebras. The theory of quiver representations may not only be viewed as a new language for a whole range of problems in linear algebra, but it also provides a platform for bringing new ideas and techniques from algebraic geometry and Lie theory into the subject. There are two major fundamental developments after Gabriel's work. In order to complete Gabriel's classification to include all Dynkin graphs, Dlab and Ringel 10] studied representations of a modulated quiver (or a species) and proved that a modulated quiver admits only finitely many indecomposable representations if and only if it is of Dynkin type. In the subsequent works [12, 31, 11, representations of both quivers and modulated quivers

Received by the editors August 14, 2003 and, in revised form, August 12, 2004.

2000 Mathematics Subject Classification. Primary 16G10, 16G20, 16G70.

This work was partially supported by the NSF of China (Grant no. 10271014), the Doctoral Program of Higher Education, and the Australian Research Council.

(C)2006 American Mathematical Society Reverts to public domain 28 years from publication 
of "tame" representation type are classified. On the other hand, by employing the methods of invariant theory, Kac [24, 25] was able to establish a connection between the indecomposable representations of any finite quiver and the positive roots of the corresponding Kac-Moody algebra with a symmetric generalized Cartan matrix. See 32 for a further developing influence of the theory of finite-dimensional algebras in the study of Lie theory.

These fundamental works provide two major approaches in the representation theory of algebras. The quiver approach over an algebraically closed field often uses geometric methods and deals with the case of symmetric (generalized) Cartan matrices in the context of Lie theory, while the modulated quiver approach, though a bit artificial, is purely algebraic, and suitable for an arbitrary ground field. In particular, the underlying valued quiver of a modulated quiver over a finite field is usually not constantly valued. So the corresponding Cartan matrix is not symmetric, but symmetrizable. Thus, the modulated quiver approach covers all symmetrizable Cartan matrices. Since a symmetrizable Cartan matrix can be obtained by folding the graph of a symmetric Cartan matrix via a graph automorphism, it is natural to ask if such an idea of folding can be lifted to the algebra and representation levels.

Originating from Galois theory, Frobenius morphisms are fundamental in the theory of algebraic groups and their representations. It is the key idea to investigating the rational structure of algebraic groups and to relating finite groups of Lie type to the corresponding algebraic groups (see 3 ). In the theory of rational representations of algebraic groups, Frobenius kernels play a key role, and the Frobenius twisting functor is centrally important in solving the main problems of the theory such as Steinberg's tensor product theorem and the Lusztig conjecture (see [22]).

In this paper, we shall combine the idea of folding graphs with the idea of Frobenius morphisms to build a direct bridge between the quiver and modulated quiver approaches. By introducing Frobenius morphisms on algebras $A$ defined over $\overline{\mathbb{F}}_{q}$, we shall prove that representations of the fixed point algebra $A^{F}$ are obtained by taking fixed points of $F$-stable representations of $A$. In particular, if $A$ is the path algebra of a quiver $Q$ which admits an admissible automorphism $\sigma$, i.e., the path algebra of an ad-quiver (see Example 3.5), and $F$ is the Frobenius morphism on $A$ induced from $\sigma$, then $A^{F}$ is isomorphic to the tensor algebra of the modulated quiver obtained by "folding" $Q$ and $A$ through $\sigma$ and $F$, respectively. Thus, the representation theory of an $\mathbb{F}_{q}$-modulated quiver can be realized as that of an ordinary quiver $Q$ by simply taking fixed points of $F$-stable representations of $Q$ over $\overline{\mathbb{F}}_{q}$. We further prove that the representation types of modulated quivers are completely determined by the corresponding classification for quivers and that every finite-dimensional hereditary (basic) algebra over a finite field is isomorphic to the fixed point algebra of a path algebra. We also establish a relation between the Auslander-Reiten theories of $A$ and its fixed point algebra $A^{F}$.

We organize the paper as follows. Section 2 is a brief introduction to $\mathbb{F}_{q^{-}}$ structures of vector spaces. In $\S 3$ we consider algebras $A$ with Frobenius morphisms $F$ and define $F$-stable $A$-modules. Then we show that the category of $F$-stable $A$-modules is equivalent to the category of modules over the fixed point algebra $A^{F}$. In $\S 4$ we define the Frobenius twist of an $A$-module and introduce the notion of $F$-periodic $A$-modules. As a result, we prove in $\S 5$ that each indecomposable $A^{F}$-module can be obtained by "folding" $F$-periodic $A$-modules. In 
particular, if $A$ is finite dimensional, there is a bijection between indecomposable $A^{F}$-modules and $F$-orbits of the indecomposable $A$-modules. As a first application to representations of quivers with automorphisms, it is shown in $\S 6$ that the representation theory of a modulated quiver (or a species) over $\mathbb{F}_{q}$ is completely determined by the representation theory of the corresponding quiver over $\overline{\mathbb{F}}_{q}$. We further prove that every finite-dimensional hereditary algebra over $\mathbb{F}_{q}$ is Morita equivalent to some $A^{F}$, where $A$ is the path algebra of a quiver $Q$ over $\overline{\mathbb{F}}_{q}$ and $F$ is the Frobenius morphism induced from an automorphism of $Q$. In $\S 7$ we establish a relation between almost split sequences of $A$ and $A^{F}$. Section 8 is devoted to proving that the Auslander-Reiten (modulated) quiver of $A^{F}$ is obtained by folding the Auslander-Reiten quiver of $A$. In $\S 9$ and $\S 10$, we present formulae for the number of indecomposable $F$-stable representations of an ad-quiver with a fixed dimension vector and generalize Kac's theorem to cover all modulated quivers and their associated (symmetrizable) Kac-Moody algebras. Thus, we reobtain and generalize some results in [18, 8, 21].

Arising from the representation theory of semi-simple Lie algebras and algebraic groups, quasi-hereditary algebras introduced by E. Cline, B. Parshall and L. Scott [33, 4] are generally regarded as a Lie theory approach to the theory of finite-dimensional algebras. The present work can also be regarded as another such example. In fact, it was motivated by a problem for the universal enveloping algebras of semi-simple Lie algebras. In [6, 7], a strong monomial basis property was discovered for quantized enveloping algebras of simply-laced Dynkin or cyclic type. To establish a similar property for a non-simply-laced Dynkin type, the theory developed in this paper will allow us to use some geometric methods from the quiver approach in the modulated quiver case. In a forthcoming paper, we shall complete our task on the strong monomial basis property for all quantized enveloping algebras of finite type.

Throughout, let $q$ be a prime power, let $\mathbb{F}_{q}$ be the finite field of $q$ elements and let $k$ be the algebraic closure $\overline{\mathbb{F}}_{q}$ of $\mathbb{F}_{q}$. For any $r \geqslant 1$, let $\mathbb{F}_{q^{r}}$ denote the unique extension field of $\mathbb{F}_{q}$ of degree $r$ contained in $k=\overline{\mathbb{F}}_{q}$. All modules considered are left modules of finite dimension over the base field. If $M$ is a module, $[M]$ denotes the class of modules isomorphic to $M$, i.e., the isoclass of $M$. For any field $k$, the notation $k^{m \times n}$ denotes the set of all $m \times n$ matrices over $k$.

\section{2. $\mathbb{F}_{q}$-STRUCTURES ON VECTOR SPACES}

An $\mathbb{F}_{q}$-structure on a vector space $V$ over $k$ is an $\mathbb{F}_{q}$-subspace $V_{0}$ of $V$ viewed as a space over $\mathbb{F}_{q}$ such that the canonical homomorphism $V_{0} \otimes_{\mathbb{F}_{q}} k \rightarrow V$ is an isomorphism. We shall always identify $V$ with $V_{0} \otimes k$ in the sequel.

Lemma 2.1. A k-space $V$ has an $\mathbb{F}_{q}$-structure $V_{0}$ if and only if

$$
V_{0}=V^{F}:=\{v \in V \mid F(v)=v\}
$$

for some $\mathbb{F}_{q}$-linear isomorphism $F: V \rightarrow V$ satisfying

(a) $F(\lambda v)=\lambda^{q} F(v)$ for all $v \in V$ and $\lambda \in k$;

(b) for any $v \in V, F^{n}(v)=v$ for some $n>0$.

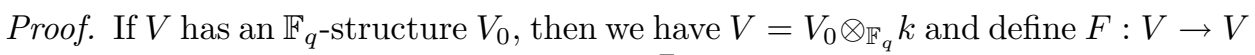
by sending $v \otimes \lambda$ to $v \otimes \lambda^{q}$. Clearly, $V_{0}=V^{F}$ and $F$ satisfies the conditions (a) and (b). The proof for the converse is given in $[9,3.5]$. 
The map $F$ is called a Frobenius map. By the lemma, we see that an $\mathbb{F}_{q}$-structure on $V$ is equivalent to the existence of a Frobenius map. From the proof we see that if $F: V \rightarrow V$ is a Frobenius map, then there is a basis $\left\{v_{i}\right\}$ of $V$ such that $F\left(\sum_{i} \lambda_{i} v_{i}\right)=\sum_{i} \lambda_{i}^{q} v_{i}$

Corollary 2.2. If $F$ and $F^{\prime}$ are Frobenius maps on a finite-dimensional space $V$, then $F^{\prime} \circ F^{-1}$ is $k$-linear on $V$ and there is a positive integer $n$ such that $F^{n}=F^{\prime n}$.

Proof. Since $V=V^{F} \otimes_{\mathbb{F}_{q}} k=V^{F^{\prime}} \otimes_{\mathbb{F}_{q}} k$ and $V$ is finite dimensional, there is a positive integer $n$ such that

$$
V\left(\mathbb{F}_{q^{n}}\right):=V^{F} \otimes_{\mathbb{F}_{q}} \mathbb{F}_{q^{n}}=V^{F^{\prime}} \otimes_{\mathbb{F}_{q}} \mathbb{F}_{q^{n}}
$$

Choose bases $\left\{v_{i}\right\}$ and $\left\{w_{i}\right\}$ for $V^{F}$ and $V^{F^{\prime}}$, respectively, and write $w_{j}=\sum_{i} x_{i j} v_{i}$ with $x_{i j} \in \mathbb{F}_{q^{n}}$. Now, one easily checks that $F^{n}=F^{\prime n}$ on $V\left(\mathbb{F}_{q^{n}}\right)$, and hence, on V.

Let $\mathcal{V}_{k, \mathbb{F}_{q}}$ be the category whose objects are vector spaces over $k$ with fixed $\mathbb{F}_{q}$-structures and whose morphisms are linear maps $f: V \rightarrow W$ defined over $\mathbb{F}_{q}$, namely, $f\left(V_{0}\right) \subseteq W_{0}$. Clearly, if $F_{V}$ and $F_{W}$ are the Frobenius maps on $V$ and $W$ defining $V_{0}$ and $W_{0}$, respectively, then $f$ is defined over $\mathbb{F}_{q}$ if and only if $F_{W} \circ f=f \circ F_{V}$. In particular, if $F$ and $F^{\prime}$ are two Frobenius maps on $V$, then any $\mathbb{F}_{q}$-linear isomorphism from $V^{F}$ to $V^{F^{\prime}}$ induces a $k$-linear isomorphism $\theta$ on $V$ such that $\theta \circ F=F^{\prime} \circ \theta$. In other words, up to isomorphism in $\mathcal{V}_{k, \mathbb{F}_{q}}$, the $\mathbb{F}_{q}$-structure on $V$ is unique. Note that $\mathcal{V}_{k, \mathbb{F}_{q}}$ is an abelian category.

We shall represent the fixed $\mathbb{F}_{q}$-structure on a $k$-space $V$ by a Frobenius map $F_{V}$. Thus, the objects $V$ in $\mathcal{V}_{k, \mathbb{F}_{q}}$ are sometimes written as $\left(V, F_{V}\right)$, and

$$
\operatorname{Hom}_{\mathcal{V}_{k, \mathbb{F}}}(V, W)=\left\{f \in \operatorname{Hom}_{k}(V, W) \mid F_{W} \circ f=f \circ F_{V}\right\} .
$$

For $\left(V, F_{V}\right),\left(W, F_{W}\right) \in \mathrm{Ob}\left(\mathcal{V}_{k, \mathbb{F}_{q}}\right)$, there is an induced $\mathbb{F}_{q}$-linear map

$$
F_{(V, W)}: \operatorname{Hom}_{k}(V, W) \rightarrow \operatorname{Hom}_{k}(V, W) ; f \mapsto F_{(V, W)}(f)=F_{W} \circ f \circ F_{V}^{-1} .
$$

Note that, when $V$ is infinite dimensional, $F_{(V, W)}$ is not necessarily a Frobenius map on $\operatorname{Hom}_{k}(V, W)$ in the sense of Lemma 2.1. However, we have the following.

Lemma 2.3. For $\left(V_{1}, F_{1}\right),\left(V_{2}, F_{2}\right) \in \mathrm{Ob}\left(\mathcal{V}_{k, \mathbb{F}_{q}}\right)$, let $F=F_{\left(V_{1}, V_{2}\right)}$ be defined as above. Then we have an $\mathbb{F}_{q}$-space isomorphism

$$
\operatorname{Hom}_{\mathbb{F}_{q}}\left(V_{1}^{F_{1}}, V_{2}^{F_{2}}\right) \cong \operatorname{Hom}_{\mathcal{V}_{k, \mathbb{F}}}\left(V_{1}, V_{2}\right)=\operatorname{Hom}_{k}\left(V_{1}, V_{2}\right)^{F}
$$

In particular, if $V_{1}$ is finite dimensional, then $F$ is a Frobenius map on $\operatorname{Hom}_{k}\left(V_{1}, V_{2}\right)$.

Proof. The isomorphism is defined by sending $f$ to $f \otimes 1$ with inverse defined by restriction. It is clear that $F$ is an $\mathbb{F}_{q}$-linear map satisfying 2.1 (a). If $V_{1}$ is finite dimensional, $F$ also satisfies 2.1 (b). Hence, $F$ is a Frobenius map.

Remarks 2.4. (1) Every Frobenius map on a finite-dimensional $k$-space $V$ induces the Frobenius map $F=F_{(V, V)}$ on the algebra $\operatorname{End}_{k}(V)$ of all linear transformations on $V$. Clearly, $F$ is an $\mathbb{F}_{q}$-algebra automorphism and induces an $\mathbb{F}_{q}$-algebra isomorphism $\operatorname{End}_{k}(V)^{F} \cong \operatorname{End}_{\mathbb{F}_{q}}\left(V^{F}\right)$.

(2) The restriction of $F$ on $\operatorname{End}_{k}(V)$ to the general linear group $G L(V)$ induces a Frobenius morphism on the connected algebraic group $G L(V)$ to which the following Lang-Steinberg theorem applies. 
Theorem 2.5 (Lang-Steinberg). Let $G$ be a connected affine algebraic group and let $F$ be a surjective endomorphism of $G$ with a finite number of fixed points. Then the map $\mathcal{L}: g \mapsto g^{-1} F(g)$ from $G$ to itself is surjective.

\section{Algebras with Frobenius morphisms}

Let $A$ be a $k$-algebra with identity 1 . We do not assume generally that $A$ is finite dimensional. A map $F_{A}: A \rightarrow A$ is called a Frobenius morphism on $A$ if it is a Frobenius map on the $k$-space $A$, and it is also an $\mathbb{F}_{q}$-algebra isomorphism sending 1 to 1 . For example, for a finite-dimensional $k$-space $V, A=\operatorname{End}_{k}(V)$ admits a Frobenius morphism $F_{(V, V)}$ induced from a Frobenius map $F_{V}$ on $V$ (see Remarks 2.4).

Given a Frobenius morphism $F_{A}$ on $A$, let

$$
A^{F}:=A^{F_{A}}=\left\{a \in A \mid F_{A}(a)=a\right\}
$$

be the set of $F_{A}$-fixed points. Then $A^{F}$ is an $\mathbb{F}_{q}$-subalgebra of $A$, and $A=A^{F} \otimes k$. The Frobenius morphism $F_{A}$ on $A$ is given by $F_{A}(a \otimes \lambda)=a \otimes \lambda^{q}$ for all $a \in A^{F}, \lambda \in$ $k$.

Let $M$ be a finite-dimensional $A$-module and let $\pi=\pi_{M}: A \rightarrow \operatorname{End}_{k}(M)$ be the corresponding representation. We say that $M$ is $F_{A^{-}}$stable (or simply $F$ stable) if there is an $\mathbb{F}_{q}$-structure $M_{0}$ of $M$ such that $\pi$ induces a representation $\pi_{0}: A^{F} \rightarrow \operatorname{End}_{\mathbb{F}_{q}}\left(M_{0}\right)$ of $A^{F}$. Clearly, by Lemma 2.1, $M$ is $F$-stable if and only if there is a Frobenius map $F_{M}: M \rightarrow M$ such that

$$
F_{M}(a m)=F_{A}(a) F_{M}(m), \text { for all } a \in A, m \in M \text {. }
$$

In terms of the corresponding representation $\pi$, the $F$-stability of $M$ simply means that there is a Frobenius map $F_{M}$ on $M$ such that $\pi \circ F_{A}=F_{(M, M)} \circ \pi$. Thus, if $M$ is an $F$-stable $A$-module with respect to $F_{M}$, then $M=M^{F} \otimes_{\mathbb{F}_{q}} k, F_{M}(m \otimes \lambda)=m \otimes \lambda^{q}$ for all $m \in M^{F}, \lambda \in k$, and $M^{F}$ is an $A^{F}$-module. Here, again for notational simplicity, we write $M^{F}$ for $\left.M^{F_{M}} 1\right]$ We shall also use, sometimes, the notation $\left(M, F_{M}\right)$ for an $F$-stable module $M$.

Lemma 3.1. Let $\left(M_{1}, F_{1}\right)$ and $\left(M_{2}, F_{2}\right)$ be two $F$-stable A-modules. Then $M_{1}^{F_{1}} \cong$ $M_{2}^{F_{2}}$ as $A^{F}$-modules if and only if $M_{1} \cong M_{2}$ as A-modules. In particular, if $M_{1}=$ $M_{2}=M$, then $M^{F_{1}} \cong M^{F_{2}}$ as $A^{F}$-modules.

Proof. Since $M_{i}=M_{i}^{F_{i}} \otimes_{\mathbb{F}_{q}} k$ and $A=A^{F} \otimes_{\mathbb{F}_{q}} k$, the lemma follows directly from the Noether-Deuring Theorem (see for example [5, p. 139]).

This result shows that, up to isomorphism, it does not matter which Frobenius maps (or $\mathbb{F}_{q}$-structures) on $M$ we shall work with when considering $F$-stable modules.

Let $\bmod ^{F}-A$ denote the category whose objects are $F$-stable modules $M=$ $\left(M, F_{M}\right)$. The morphisms from $\left(M, F_{M}\right)$ to $\left(N, F_{N}\right)$ are given by homomorphisms $\theta \in \operatorname{Hom}_{A}(M, N)$ such that $\theta \circ F_{M}=F_{N} \circ \theta$, that is, $A$-module homomorphisms compatible with their $\mathbb{F}_{q}$-structures. It is easy to see that $\bmod ^{F}-A$ is an abelian $\mathbb{F}_{q}$-category.

The next result allows us to embed a module category defined over a finite field into a category defined over the algebraic closure of the finite field.

\footnotetext{
${ }^{1}$ It should be understood that the $F^{\prime}$ 's in $A^{F}$ and $M^{F}$ are not the same.
} 
Theorem 3.2. The abelian category $\bmod ^{F}-A$ is equivalent to the category $\bmod -A^{F}$ of finite-dimensional $A^{F}$-modules.

Proof. Let $M=\left(M, F_{M}\right)$ be an object in $\bmod ^{F}-A$. We define $\Phi(M):=M^{F}$ which is an $A^{F}$-module. Now let $\theta:\left(M_{1}, F_{2}\right) \rightarrow\left(M_{2}, F_{2}\right)$ be a morphism in $\bmod ^{F}-A$. Since $\theta \circ F_{1}=F_{2} \circ \theta, \theta$ induces a map $\Phi(\theta): M_{1}^{F_{1}} \rightarrow M_{2}{ }^{F_{2}}$, which is obviously an $A^{F}$-module homomorphism. This gives a functor $\Phi: \bmod ^{F}-A \rightarrow \bmod -A^{F}$.

Conversely, for each $A^{F}$-module $X$, we set $\Psi(X)=X \otimes_{\mathbb{F}_{q}} k$ and define a Frobenius map

$$
F_{\Psi(X)}: \Psi(X) \longrightarrow \Psi(X) ; x \otimes \lambda \longmapsto x \otimes \lambda^{q} .
$$

By defining $(a \otimes \lambda)(x \otimes \mu)=a x \otimes \lambda \mu$ for $a \otimes \lambda \in A^{F} \otimes k=A$ and $x \otimes \mu \in \Psi(X)$ and noting $F_{A}(a \otimes \lambda)=a \otimes \lambda^{q}$, we obtain an $A$-module structure on $\Psi(X)$, which is clearly $F$-stable. Further, for any morphism $f: X_{1} \rightarrow X_{2}$ in mod- $A^{F}$, the map

$$
\Psi(f)=f \otimes 1: \Psi\left(X_{1}\right) \longrightarrow \Psi\left(X_{2}\right)
$$

is obviously an $A$-module homomorphism satisfying $\Psi(f) \circ F_{1}=F_{2} \circ \Psi(f)$, where $F_{i}=F_{\Psi\left(X_{i}\right)}$. Hence, we obtain a functor $\Psi: \bmod -A^{F} \rightarrow \bmod ^{F}-A$.

From the construction, we easily see that

$$
\Psi \Phi \cong 1_{\bmod ^{F}-A} \text { and } \Phi \Psi \cong 1_{\bmod -A^{F}},
$$

where $1_{\bmod ^{F}-A}$ and $1_{\bmod -A^{F}}$ denote the identity functors of $\bmod ^{F}-A$ and $\bmod -A^{F}$, respectively.

Corollary 3.3. There is a one-to-one correspondence between isoclasses of indecomposable $A^{F}$-modules and isoclasses of indecomposable F-stable A-modules.

Let $\left(M, F_{M}\right)$ be an $F$-stable $A$-module. For each submodule $N$ of $M$ (not necessarily an $F_{M}$-stable subspace), the image $F_{M} N$ is an $A$-submodule of $M$.

Proposition 3.4. Let $\left(M, F_{M}\right)$ and $\left(N, F_{N}\right)$ be two F-stable modules.

(a) Every submodule $M^{\prime}$ of $M$ which is also an $F_{M}$-stable subspace is an $F$ stable A-module. In particular, both the radical $\operatorname{Rad} M$ and socle $\operatorname{Soc} M$ of $M$ are $F$-stable modules.

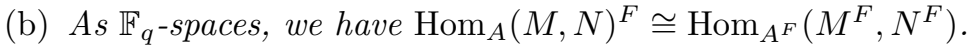

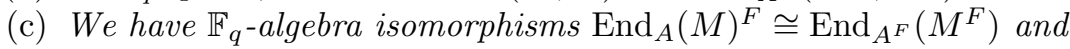

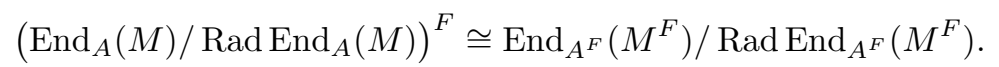

Proof. (a) Since $F_{M} M^{\prime}=M^{\prime}$, the restriction of $F_{M}$ to $M^{\prime}$ defines a Frobenius map on $M^{\prime}$. So $M^{\prime}$ is an $F$-stable module, as the condition (3.0.1) is automatically satisfied. If $S$ is a maximal (resp. simple) submodule of $M$, so is $F_{M} S$. Thus, both $\operatorname{Rad} M$ and Soc $M$ are submodules which are $F_{M}$-stable (subspaces) and hence are $F$-stable modules.

(b) This is a consequence of the category equivalence given in Theorem 3.2 ,

(c) The first statement follows from (b) and Lemma 2.3. We now prove the last isomorphism. We first observe that if $B$ is a semisimple algebra with Frobenius morphism $F_{B}$, then the fixed point algebra $B^{F}$ is also semisimple. Since

$$
\left(\operatorname{End}_{A}(M) / \operatorname{Rad}_{\operatorname{End}}(M)\right)^{F} \cong\left(\operatorname{End}_{A}(M)\right)^{F} /\left(\operatorname{Rad} \operatorname{End}_{A}(M)\right)^{F},
$$

it remains to prove that

$$
\left(\operatorname{Rad}_{\operatorname{End}}(M)\right)^{F}=\operatorname{Rad}\left(\operatorname{End}_{A}(M)\right)^{F} .
$$


The inclusion " $\supseteq$ " follows from the semisimplicity of the right-hand side of the above isomorphism, while the inverse inclusion " $\subseteq$ " follows from the fact that $\left(\operatorname{Rad} \operatorname{End}_{A}(M)\right)^{F}$ is nilpotent.

An $A$-module $M$ is called $F$-periodic if there exists an $F$-stable $A$-module $N$ such that $M$ is isomorphic to a direct summand of $N$ (denoted by $M \mid N$ ). We shall see in the next section that for a finite-dimensional algebra $A$ with Frobenius morphism $F_{A}$, every $A$-module is $F$-periodic. However, in Example 4.7, we shall see that, for an infinite-dimensional algebra, there are modules which are not $F$-periodic.

We end this section with the following example which is important in $\S \S 6-10$.

Example 3.5. Let $Q=\left(Q_{0}, Q_{1}\right)$ be a finite quiver without loops, where $Q_{0}$ resp. $Q_{1}$ denotes the set of vertices resp. arrows of $Q$. For each arrow $\rho$ in $Q_{1}$, we denote by $h \rho$ and $t \rho$ the head and the tail of $\rho$, respectively. Let $\sigma$ be an automorphism of $Q$, that is, $\sigma$ is a permutation on the vertices of $Q$ and on the arrows of $Q$ such that $\sigma(h \rho)=h \sigma(\rho)$ and $\sigma(t \rho)=t \sigma(\rho)$ for any $\rho \in Q_{1}$. We further assume, following [29, 12.1.1], that $\sigma$ is admissible, that is, there are no arrows connecting vertices in the same orbit of $\sigma$ in $Q_{0}$. We shall call the pair $(Q, \sigma)$ an admissible quiver, or simply an ad-quiver.

Let $A:=k Q$ be the path algebra of $Q$ over $k=\overline{\mathbb{F}}_{q}$ which has identity $1=$ $\sum_{i \in Q_{0}} e_{i}$, where $e_{i}$ is the idempotent (or the length 0 path) corresponding to the vertex $i$. Then $\sigma$ induces a Frobenius morphism (cf. Lemma 2.1)

$$
F_{Q, \sigma}=F_{Q, \sigma ; q}: A \rightarrow A ; \sum_{s} x_{s} p_{s} \longmapsto \sum_{s} x_{s}^{q} \sigma\left(p_{s}\right),
$$

where $\sum_{s} x_{s} p_{s}$ is a $k$-linear combination of paths $p_{s}$, and $\sigma\left(p_{s}\right)=\sigma\left(\rho_{t}\right) \cdots \sigma\left(\rho_{1}\right)$ if $p_{s}=\rho_{t} \cdots \rho_{1}$ for arrows $\rho_{1}, \ldots, \rho_{t}$ in $Q_{1}$. We shall investigate the structure of $A^{F}$ in $\S 6$.

\section{Twisting modules With Frobenius maps}

Let $M$ be an $A$-module and let $F_{M}: M \rightarrow M$ be a Frobenius map on the space $M$. Note that $M$ is not necessarily $F$-stable. We define its (external) Frobenius twist (with respect to the Frobenius map $F_{M}$ ) to be the $A$-module $M^{\left[F_{M}\right]}$ such that $M^{\left[F_{M}\right]}=M$ as vector spaces with $F$-twisted action

$$
a * m:=F_{M}\left(F_{A}^{-1}(a) F_{M}^{-1}(m)\right) \text { for all } a \in A, m \in M .
$$

If $\pi: A \rightarrow \operatorname{End}_{k}(M)$ and $\pi^{\left[F_{M}\right]}: A \rightarrow \operatorname{End}_{k}\left(M^{\left[F_{M}\right]}\right)$ denote the corresponding representations, then

$$
\pi^{\left[F_{M}\right]}(a)=F_{(M, M)}\left(\pi\left(F_{A}^{-1}(a)\right)\right)=F_{M} \circ \pi\left(F_{A}^{-1}(a)\right) \circ F_{M}^{-1} \text { for all } a \in A,
$$

where $F_{(M, M)}$ is the induced Frobenius map on $\operatorname{End}_{k}(M)$ (cf. Remarks 2.4).

Lemma 4.1. Up to isomorphism, the Frobenius twist $M^{\left[F_{M}\right]}$ is independent of the choice of the Frobenius map $F_{M}$ on $M$.

Proof. If $F_{M}$ and $F_{M}^{\prime}$ are two Frobenius maps on $M$, then the linear isomorphism $f:=F_{M}^{\prime} \circ F_{M}^{-1}: M \rightarrow M$ is clearly an $A$-module isomorphism from $M^{\left[F_{M}\right]}$ to $M^{\left[F_{M}^{\prime}\right]}$. 
By the lemma, we shall denote $M^{\left[F_{M}\right]}$ and $\pi^{\left[F_{M}\right]}$ by $M^{[1]}$ and $\pi^{[1]}$, respectively. Similarly, we define $M^{[-1]}$ to be the $A$-module given by $\pi^{[-1]}: A \rightarrow \operatorname{End}_{k}(M)$, where

$$
\pi^{[-1]}(a)=F_{M}^{-1} \circ \pi\left(F_{A}(a)\right) \circ F_{M} \text { for all } a \in A .
$$

Inductively, for each integer $s>1$, we define $M^{[s]}=\left(M^{[s-1]}\right)^{[1]}$ and $M^{[-s]}=$ $\left(M^{[-s+1]}\right)^{[-1]}$ with respect to the same given $F_{M}$.

Proposition 4.2. The Frobenius twist ( $)^{[1]}$ defines a category equivalence from mod- $A$ onto itself. In particular, if $M$ and $N$ are two A-modules, then for any



Proof. Given two $A$-modules $M$ and $N$ with Frobenius maps $F_{M}$ and $F_{N}$, respectively, and an $A$-module homomorphism $f: M \rightarrow N$, it can be checked by using the corresponding representations that the linear map $F_{(M, N)}(f)$ defined before Lemma 2.3 is in fact an $A$-module homomorphism from $M^{[1]}$ to $N^{[1]}$. We denote this morphism by $f^{[1]}$. Thus, we obtain a functor ()$^{[1]}: \bmod -A \rightarrow \bmod -A$. This functor is clearly invertible with inverse ()$^{[-1]}$.

If $\left(M, F_{M}\right)$ is an $F$-stable $A$-module and $N$ is a submodule of $M$ (not necessarily an $F_{M}$-stable space), then the $A$-submodule $F_{M} N$ of $M$ is called the "internal" Frobenius twist of $N$. Note that $F_{M} N$ is isomorphic to the (external) Frobenius twist $N^{[1]}$ of $N$ with respect to any given Frobenius map $F_{N}$ on $N$. This is deduced from the fact that the $k$-linear map $\varphi=\left.F_{M}\right|_{N} \circ F_{N}^{-1}: N^{[1]} \rightarrow F_{M} N$ is an $A$-module isomorphism.

Proposition 4.3. Let $M$ be an A-module with a Frobenius map $F_{M}$ and let $M^{[1]}$ denote the Frobenius twist of $M$ with respect to $F_{M}$.

(a) $\left(M, F_{M}\right)$ is F-stable if and only if, as A-modules, $M^{[1]}=M$.

(b) $M^{[1]} \cong M$ if and only if there exists a Frobenius map $F_{M}^{\prime}$ on $M$ such that $M$ is $F$-stable with respect to $F_{M}^{\prime}$, that is, $M^{\left[F_{M}^{\prime}\right]}=M$ as A-modules.

Proof. The statement (a) follows directly from the definition, since $\left(M, F_{M}\right)$ is $F$ stable if and only if $\pi \circ F_{A}=F_{(M, M)} \circ \pi$, which is equivalent to $\pi=\pi^{\left[F_{M}\right]}$.

We now prove (b). Since $M^{[1]} \cong M$, there exists an $f \in G L(M)$ such that $f \circ \pi^{\left[F_{M}\right]}(a)=\pi(a) \circ f$ for all $a \in A$. By Lang-Steinberg's theorem, there exists $g \in G L(M)$ such that $f=g^{-1} \circ F(g)$, where

$$
F: G L(M) \longrightarrow G L(M), h \longmapsto F_{M} \circ h \circ F_{M}^{-1}
$$

is induced from $F_{M}$. Let $F_{M}^{\prime}=g^{-1} \circ F_{M} \circ g: M \rightarrow M$. Then $F_{M}^{\prime}$ is a Frobenius map on $M$ and, for all $a \in A$, we have

$$
\begin{aligned}
\pi^{\left[F_{M}^{\prime}\right]}(a) & =F_{M}^{\prime} \circ \pi\left(F_{A}^{-1}(a)\right) \circ\left(F_{M}^{\prime}\right)^{-1}=g^{-1} \circ F_{M} \circ g \circ \pi\left(F_{A}^{-1}(a)\right) \circ g^{-1} \circ F_{M}^{-1} \circ g \\
& =f \circ F_{M} \circ \pi\left(F_{A}^{-1}(a)\right) \circ F_{M}^{-1} \circ f^{-1}=f \circ \pi^{\left[F_{M}\right]}(a) \circ f^{-1}=\pi(a),
\end{aligned}
$$

that is, $M^{\left[F_{M}^{\prime}\right]}=M$ as $A$-modules.

We now characterize $F$-periodic modules defined at the end of last section by Frobenius twisting.

Corollary 4.4. An A-module $M$ is F-periodic if and only if $M^{[r]} \cong M$ for some integer $r$. 
Proof. Suppose $M$ is $F$-periodic. Then so is every direct summand of $M$. Since $M^{[r]} \cong M$ implies $M^{[r s]} \cong M$ for all $s \geqslant 1$, it suffices to prove the case when $M$ is indecomposable.

If $M$ is $F$-periodic and indecomposable, then there is an $F$-stable $A$-module $\left(N, F_{N}\right)$ such that $M \mid N$. Thus, $F_{N}^{n} M \mid N$ for all $n \geqslant 1$. By the Krull-RemakSchmidt theorem, there must be an integer $r \geqslant 1$ such that $F_{N}^{r} M \cong M$, i.e., $M^{[r]} \cong M$.

Conversely, suppose $M^{[r]} \cong M$. Then

$$
N:=M \oplus M^{[1]} \oplus \cdots \oplus M^{[r-1]}
$$

satisfies that $N^{[1]} \cong N$. By Proposition $4.3(\mathrm{~b}), N$ is $F$-stable with respect to a Frobenius map, thus $M$ is $F$-periodic.

Remarks 4.5. (1) For an $F$-periodic $A$-module $M$, let $r$ be the minimal integer such that $M^{[r]} \cong M$. We shall call $r$ the $F$-period of $M$, denoted by $p(M)=p_{F}(M)$. Clearly, if $M^{[s]} \cong M$, then $p(M) \mid s$ and, by Lemma 4.1 $p(M)$ is independent of the choice of the Frobenius map on $M$.

(2) Using a similar argument as in the proof of Proposition 4.3(b), for an Fperiodic $A$-module $M$ with $F$-period $r$, there is a Frobenius map $F_{M}$ on $M$ such that $M^{[r]}=M$ as $A$-modules, where $M^{[r]}$ is defined with respect to $F_{M}$.

Proposition 4.6. Let $A$ be finite dimensional. Then every A-module is F-periodic.

Proof. Let $M$ be an $A$-module with a Frobenius map $F=F_{M}$. By Lemma 2.1 there are $k$-basis $\left\{a_{1}, a_{2}, \ldots, a_{s}\right\}$ of $A$ and $k$-basis $\left\{m_{1}, m_{2}, \ldots, m_{t}\right\}$ of $M$ such that

$$
F_{A}\left(\sum_{i=1}^{s} x_{i} a_{i}\right)=\sum_{i=1}^{s} x_{i}^{q} a_{i} \text { and } F\left(\sum_{j=1}^{t} y_{j} m_{j}\right)=\sum_{j=1}^{t} y_{j}^{q} m_{j}
$$

for all $a=\sum_{i=1}^{s} x_{i} a_{i} \in A$ and all $m=\sum_{j=1}^{t} y_{j} m_{j}$ in $M$. Write $a_{i} m_{j}=\sum_{l=1}^{t} z_{i j l} m_{l}$ with $z_{i j l} \in k$. Since $k=\overline{\mathbb{F}}_{q}$, there is an integer $n$ such that all $z_{i j l}$ 's lie in $\mathbb{F}_{q^{n}}$. Thus, $F^{n}\left(a_{i} m_{j}\right)=a_{i} m_{j}$ for all $i$ and $j$. Consequently, we have

$$
\begin{aligned}
F^{n}(a m) & =\sum_{i, j} F^{n}\left(x_{i} y_{j} a_{i} m_{j}\right)=\sum_{i, j} x_{i}^{q^{n}} y_{j}^{q^{n}} F^{n}\left(a_{i} m_{j}\right) \\
& =\left(\sum_{i=1}^{s} x_{i}^{q^{n}} a_{i}\right)\left(\sum_{j=1}^{t} y_{j}^{q^{n}} m_{j}\right)=F_{A}^{n}(a) F^{n}(m) .
\end{aligned}
$$

This implies that $M^{[n]}=M$ as $A$-modules. Hence $M$ is $F$-periodic.

Recall that, for a path algebra $A$ of a quiver $Q$, an $A$-module can be identified as a representation $(V, \phi)$ of $Q$, where $V=\left\{V_{i}\right\}_{i \in Q_{0}}$ is a set of finite-dimensional vector spaces $V_{i}$ and $\phi=\left\{\phi_{\rho}\right\}_{\rho \in Q_{1}}$ is a set of linear transformations $\phi_{\rho}: V_{t \rho} \rightarrow V_{h \rho}$.

Example 4.7. Let $Q$ be the quiver with two vertices 1 and 2 and with infinitely many arrows from 1 to 2 indexed by $\rho_{i j}$ for all $i \geqslant 1$ and $1 \leqslant j \leqslant i$. That is, they are

$$
\rho_{11}, \rho_{21}, \rho_{22}, \rho_{31}, \rho_{32}, \rho_{33}, \ldots \text {. }
$$

Then the path algebra $A=k Q$ is infinite dimensional and has the identity $1=e_{1}+$ $e_{2}$. Consider the automorphism $\sigma$ of $Q$ fixing two vertices and cyclicly permuting each subset $\left\{\rho_{i j} \mid 1 \leqslant j \leqslant i\right\}$ of arrows for each fixed $i \geqslant 1$. Then $\sigma$ induces a 
Frobenius morphism $F=F_{Q, \sigma}$ on $A$ (see Example 3.5). Define a representation $V$ such that $V_{1}=V_{2}=k$ and that $\phi_{\rho_{i 1}}$ is the identity on $k$, but $\phi_{\rho_{i j}}=0$ for all $j \neq 1$. Then $V$ is two dimensional and $V^{[r]} \neq V$ for all $r \geqslant 0$. Therefore, $V$ is not $F$-periodic.

\section{Indecomposable $F$-STABle Modules}

Let $A$ be a $k$-algebra with a fixed Frobenius morphism $F_{A}$. The proof of Corollary 4.4 actually suggests a construction of indecomposable $F$-stable modules from $F$ periodic indecomposable ones.

Let $M$ be $F$-periodic with $F$-period $r=p(M)$. In view of Remarks 4.5(2), there is a Frobenius map $F_{M}$ on $M$ such that $M^{[r]}=M$ as $A$-modules. Thus, by Proposition 4.2, $M, M^{[1]}, \ldots, M^{[r-1]}$ are pairwise non-isomorphic. Let

$$
\tilde{M}=M \oplus M^{[1]} \oplus \cdots \oplus M^{[r-1]}
$$

and define a Frobenius map $F_{\tilde{M}}: \tilde{M} \rightarrow \tilde{M}$ by

$$
F_{\tilde{M}}\left(x_{0}, x_{1}, \ldots, x_{r-1}\right)=\left(F_{M}\left(x_{r-1}\right), F_{M}\left(x_{0}\right), \ldots, F_{M}\left(x_{r-2}\right)\right) .
$$

Then $\left(\tilde{M}, F_{\tilde{M}}\right)$ is $F$-stable. Thus, $\tilde{M}^{F}:=\tilde{M}^{F_{\tilde{M}}}$ is an $A^{F}$-module. By Lemma 3.1 we infer that, up to isomorphism, $\tilde{M}^{F}$ is independent of the choice of $F_{M}$.

The following result generalizes Kac's result [24, Lemma 3.4] for the path algebras of quivers.

Theorem 5.1. Maintain the notation above. Let $M$ be an $F$-periodic indecomposable A-module with $F$-period $r$. Then $\left(\tilde{M}, F_{\tilde{M}}\right)$ is indecomposable in $\bmod ^{F}-A$ and

$$
\operatorname{End}_{A^{F}}\left(\tilde{M}^{F}\right) / \operatorname{Rad}\left(\operatorname{End}_{A^{F}}\left(\tilde{M}^{F}\right)\right) \cong \mathbb{F}_{q^{r}} .
$$

Moreover, every indecomposable $A^{F}$-module is isomorphic to a module of the form $\tilde{M}^{F}$ for some F-periodic indecomposable module $M$.

Proof. The Frobenius map $F_{\tilde{M}}: \tilde{M} \rightarrow \tilde{M}$ induces a Frobenius map $\tilde{F}=F_{(\tilde{M}, \tilde{M})}$ on $\operatorname{End}_{A}(\tilde{M})$. By Proposition $3.4(\mathrm{c})$, we have an $\mathbb{F}_{q}$-algebra isomorphism

$$
\operatorname{End}_{A^{F}}\left(\tilde{M}^{F}\right) / \operatorname{Rad}\left(\operatorname{End}_{A^{F}}\left(\tilde{M}^{F}\right)\right) \cong\left(\operatorname{End}_{A}(\tilde{M}) / \operatorname{Rad}\left(\operatorname{End}_{A}(\tilde{M})\right)\right)^{\tilde{F}} .
$$

For each $f \in \operatorname{End}_{A}(\tilde{M})$, we write $f$ in matrix form as

$$
f=\left(f_{j i}\right)_{r \times r}: \tilde{M}=\bigoplus_{i=0}^{r-1} M^{[i]} \longrightarrow \bigoplus_{i=0}^{r-1} M^{[i]}=\tilde{M}
$$

where $f_{j i}: M^{[i]} \rightarrow M^{[j]}$. Then $\tilde{F}(f)=\left(g_{j i}\right)$ with $g_{j i}=f_{j-1, i-1}^{[1]}$, where the indices are integers modulo $r$. In particular, if $\tilde{F}(f)=f$, i.e., $f \in \operatorname{End}_{A}(\tilde{M})^{\tilde{F}}$, then $f_{j i}=f_{j-1, i-1}^{[1]}$ for all $0 \leqslant i, j \leqslant r-1$. Since $M, M^{[1]}, \ldots, M^{[r-1]}$ are pairwise non-isomorphic indecomposable $A$-modules, we have an algebra isomorphism

$$
\begin{aligned}
& \operatorname{End}_{A}(\tilde{M}) / \operatorname{Rad}\left(\operatorname{End}_{A}(\tilde{M})\right) \longrightarrow \prod_{i=0}^{r-1} \operatorname{End}_{A}\left(M^{[i]}\right) / \operatorname{Rad}\left(\operatorname{End}_{A}\left(M^{[i]}\right)\right) \\
& \bar{f}=\left(\bar{f}_{j i}\right) \longmapsto\left(\bar{f}_{00}, \bar{f}_{11}, \ldots, \bar{f}_{r-1, r-1}\right) .
\end{aligned}
$$


Since $\operatorname{End}_{A}\left(M^{[i]}\right) / \operatorname{Rad}\left(\operatorname{End}_{A}\left(M^{[i]}\right)\right) \cong k$ for each $0 \leqslant i \leqslant r-1$, we obtain

$$
\operatorname{End}_{A}(\tilde{M}) / \operatorname{Rad}\left(\operatorname{End}_{A}(\tilde{M})\right) \cong \underbrace{k \times \cdots \times k}_{r}=: U .
$$

The Frobenius map $\tilde{F}$ on the left-hand side induces a Frobenius map $\tilde{F}$ on $U$ given by

$$
\tilde{F}\left(x_{0}, x_{1}, \ldots, x_{r-1}\right)=\left(x_{r-1}^{q}, x_{0}^{q}, \ldots, x_{r-2}^{q}\right) .
$$

Hence,

$$
\operatorname{End}_{A^{F}}\left(\tilde{M}^{F}\right) / \operatorname{Rad}\left(\operatorname{End}_{A^{F}}\left(\tilde{M}^{F}\right)\right) \cong U^{\tilde{F}} \cong \mathbb{F}_{q^{r}} .
$$

Conversely, let $X$ be an indecomposable $A^{F}$-module such that

$$
\operatorname{End}_{A^{F}}(X) / \operatorname{Rad}\left(\operatorname{End}_{A^{F}}(X)\right) \cong \mathbb{F}_{q^{r}} .
$$

Then $X_{k}:=X \otimes_{\mathbb{F}_{q}} k$ is an $F$-stable $A$-module with the Frobenius map $F:=F_{X_{k}}$ defined by

$$
F(x \otimes \lambda)=x \otimes \lambda^{q} \text { for } x \otimes \lambda \in X \otimes_{\mathbb{F}_{q}} k .
$$

Moreover, we have a decomposition

$$
X_{k}=M_{1} \oplus \cdots \oplus M_{r}
$$

where $M_{1}, \ldots, M_{r}$ are pairwise non-isomorphic indecomposable $A$-modules. The $F_{A}$-stability of $X_{k}$ implies that

$$
F M_{1} \oplus \cdots \oplus F M_{r}=F X_{k}=X_{k}=M_{1} \oplus \cdots \oplus M_{r} .
$$

Thus, the set $\Omega:=\left\{M_{1}, \ldots, M_{r}\right\}$ is $F$-stable (up to isomorphism). We claim that $\Omega$ contains only one $F$-orbit. Let $\Omega_{1}, \ldots, \Omega_{m}$ be the orbits of $\Omega$. For each $1 \leqslant j \leqslant m$, let

$$
L_{j}=\bigoplus_{N \in \Omega_{j}} N
$$

Then

$$
L_{j} \cong N_{j} \oplus F N_{j} \oplus \cdots \oplus F^{r_{j}-1} N_{j}
$$

where $N_{j} \in \Omega_{j}$ and $r_{j}=\left|\Omega_{j}\right|$. Note that $F^{r_{j}} N_{j} \cong N_{j}$. Let $F_{j}$ be a Frobenius map on $N_{j}$. Then $F N_{j}$ is isomorphic to the Frobenius twist $N_{j}^{[1]}$ of $N_{j}$ with respect to $F_{j}$. Hence, we have $N_{j}^{\left[r_{j}\right]} \cong N_{j}$ and

$$
L_{j} \cong N_{j} \oplus N_{j}^{[1]} \oplus \cdots \oplus N_{j}^{\left[r_{j}-1\right]} \cong L_{j}^{[1]} .
$$

Then, by Proposition 4.3(b), there is a Frobenius map $F_{j}^{\prime}$ on $L_{j}$ such that $L_{j}$ is $F$-stable with respect to $F_{j}^{\prime}$. These Frobenius maps $F_{j}^{\prime}$ induce a Frobenius map $F^{\prime}$ on $\bigoplus_{j=1}^{m} L_{j}$ defined by

$$
F^{\prime}\left(v_{1}, \ldots, v_{m}\right)=\left(F_{1}^{\prime}\left(v_{1}\right), \ldots, F_{m}^{\prime}\left(v_{m}\right)\right) .
$$

By Lemma 3.1 the isomorphism $X_{k} \cong \bigoplus_{j=1}^{m} L_{j}$ implies that

$$
X=X_{k} F \cong\left(\bigoplus_{j=1}^{m} L_{j}\right)^{F^{\prime}}=\bigoplus_{j=1}^{m} L_{j}^{F_{j}^{\prime}} .
$$

Since $X$ is indecomposable, we must have $m=1$ and the required isomorphism

$$
X_{k} \cong L_{1} \cong N_{1} \oplus N_{1}^{[1]} \oplus \cdots \oplus N_{1}^{[r-1]} \text {. }
$$


From the proof, we obtain the following correspondence.

Corollary 5.2. If $A$ is finite dimensional, then there is a one-to-one correspondence between the isoclasses of indecomposable $A^{F}$-modules and the F-orbits of the isoclasses of indecomposable A-modules.

A finite-dimensional algebra $B$ over a field is called representation-finite if there are only finitely many isoclasses of (finite-dimensional) indecomposable $B$-modules. Theorem 5.1 and Proposition 4.6 imply immediately the following (cf. [15] and 23]).

Corollary 5.3. Let $A$ be finite dimensional and let $F_{A}$ be a Frobenius morphism on $A$. Then, $A$ is representation-finite if and only if $A^{F}$ is also.

An $A^{F}$-module $X$ is called absolutely indecomposable if $X \otimes_{\mathbb{F}_{q}} k$ is an indecomposable $A$-module.

Corollary 5.4. An $A^{F}$-module $X$ is absolutely indecomposable if and only if there is an F-stable indecomposable A-module $M=(M, F)$ such that $X \cong M^{F}$.

\section{Finite-dimensional hereditary algebras}

The first application of our theory is to show that every finite-dimensional hereditary (basic) algebra over a finite field is isomorphic to the $F$ fixed point algebra of the path algebra of a finite ad-quiver (see Example 3.5). Thus, the representation theory of a finite dimensional hereditary algebra is completely determined by the counterpart of the corresponding ad-quivers.

We first recall the notion of modulated quivers (cf. [10] and [2, 4.1.9]).

Definition 6.1. A valued graph is a graph without loops together with a positive integer $d_{x}$ for each vertex $x$ and a pair of positive integers $\left({ }_{x} c_{y}^{\gamma},{ }_{y} c_{x}^{\gamma}\right)$ for each edge $x \frac{\gamma}{y}$ satisfying ${ }_{x} c_{y}^{\gamma} d_{y}={ }_{y} c_{x}^{\gamma} d_{x}$. A valued graph together with an orientation is called a valued quiver, and a valued quiver is called simple if it has no parallel arrows. (Thus, opposite arrows between two vertices are allowed in a simple-valued quiver.) A modulation ${ }^{2} \mathbb{M}$ of a valued quiver consists of an assignment of a division ring $D_{x}$ to each vertex $x$, and a $D_{y}$ - $D_{x}$-bimodule $M_{\rho}$ to each arrow $\rho: x \longrightarrow y$ with the underlying edge $x \frac{\gamma}{y}$ satisfying

(1) $\operatorname{Hom}_{D_{y}}\left(M_{\rho}, D_{y}\right) \cong \operatorname{Hom}_{D_{x}}\left(M_{\rho}, D_{x}\right)$,

(2) $\operatorname{dim}_{D_{y}}\left(M_{\rho}\right)={ }_{x} c_{y}^{\gamma}, \operatorname{dim}_{D_{x}}\left(M_{\rho}\right)={ }_{y} c_{x}^{\gamma}$.

Finally, a modulated quiver $\mathcal{Q}=(\Gamma, \mathbb{M})$ consists of a valued quiver $\Gamma$ and a modulation $\mathbb{M}$ (of $\Gamma$ ). In case all the division rings $D_{x}$ contain a common central field $K$ such that the dimensions of all $D_{x}$ over $K$ are finite and that $K$ operates centrally on each bimodule $M_{\rho}$, we call $\mathcal{Q}$ a $K$-modulated quiver or a $K$-species (cf. [15] and [10]).

A modulated quiver is simple if its underlying quiver is simple.

Let $\mathcal{Q}=(\Gamma, \mathbb{M})$ be a modulated quiver with $\Gamma_{0}$ (resp. $\left.\Gamma_{1}\right)$ the set of vertices (resp. arrows) of $\Gamma$ and $\mathbb{M}=\left(\left\{D_{x}\right\}_{x \in \Gamma_{0}},\left\{M_{\rho}\right\}_{\rho \in \Gamma_{1}}\right)$. Let $R=\bigoplus_{x \in \Gamma_{0}} D_{x}$ and $M=\bigoplus_{\rho \in \Gamma_{1}} M_{\rho}$. Then $M$ is a natural $R$ - $R$-bimodule. The $R$-algebra

$$
T(\mathcal{Q}):=\bigoplus_{n \geqslant 0} M^{\otimes n}, \quad \text { where } M^{\otimes 0}=R, M^{\otimes n}=\underbrace{M \otimes_{R} \cdots \otimes_{R} M}_{n},
$$

\footnotetext{
If we follow the definition given in $\left[2\right.$ 4.1.9], then $M_{\rho}= \begin{cases}x M_{y}^{\gamma}, & \text { if } \rho=y \stackrel{\gamma}{\longrightarrow} x, \\ y M_{x}^{\gamma}, & \text { if } \rho=x \stackrel{\gamma}{\longrightarrow} y .\end{cases}$
} 
is called the path (or tensor) algebra of $\mathcal{Q}$. Thus, a non-zero tensor $x_{n} \otimes \cdots \otimes x_{1}$ with $x_{i} \in M_{\rho_{i}}$ implies that $\rho_{n} \cdots \rho_{1}$ is a path in $\Gamma$.

For a modulated quiver $\mathcal{Q}=(\Gamma, \mathbb{M})$, let $\overline{\mathcal{Q}}=(\bar{\Gamma}, \overline{\mathbb{M}})$ be the associated simple modulated quiver obtained by summing up the valuations and bimodules over parallel arrows. More precisely, $\bar{\Gamma}$ is a simple-valued quiver defined by setting $\bar{\Gamma}_{0}=\Gamma$, $\bar{\Gamma}_{1}=\{\bar{\rho}: x \rightarrow y\}$, where $\bar{\rho}:=\{\rho \in \Gamma \mid t \rho=x, h \rho=y\}$, and setting the valuation for the arrow $\bar{\rho}: x \rightarrow y$ to be $\left(c_{\bar{\rho}}, c_{\bar{\rho}}^{\prime}\right)$, where $c_{\bar{\rho}}=\sum_{\rho \in \bar{\rho}} x c_{y}^{\rho}$ and $c_{\bar{\rho}}^{\prime}=\sum_{\rho \in \bar{\rho}} y c_{x}^{\rho}$. The modulation $\overline{\mathbb{M}}=\left(\left\{D_{x}\right\}_{x \in \bar{\Gamma}_{0}},\left\{M_{\bar{\rho}}\right\}_{\bar{\rho} \in \bar{\Gamma}_{1}}\right)$ is defined by setting $M_{\bar{\rho}}=\bigoplus_{\rho \in \bar{\rho}} M_{\rho}$.

Definition 6.2. Let $\mathcal{Q}=(\Gamma, \mathbb{M})$ and $\mathcal{Q}^{\prime}=\left(\Gamma^{\prime}, \mathbb{M}^{\prime}\right)$ be two modulated quivers. We say that $\mathcal{Q} \cong \mathcal{Q}^{\prime}$ if there exists a quiver isomorphism $\tau: \bar{\Gamma} \stackrel{\sim}{\longrightarrow} \overline{\Gamma^{\prime}}$ such that (1) $D_{x} \cong D_{\tau(x)}^{\prime}$ as division rings, and (2) $M_{\bar{\rho}} \cong M_{\tau(\bar{\rho})}^{\prime}$ as bimodules via (1).

Clearly, if $\mathcal{Q} \cong \mathcal{Q}^{\prime}$, then we have algebra isomorphism $T(\mathcal{Q}) \cong T\left(\mathcal{Q}^{\prime}\right)$.

We now construct a modulated quiver from an ad-quiver. Given a finite ad-quiver $(Q, \sigma)$, let $I=\Gamma_{0}$ and $\Gamma_{1}$ denote the set of $\sigma$-orbits in $Q_{0}$ and $Q_{1}$, respectively. Thus, we obtain a new quiver $\Gamma=\left(\Gamma_{0}, \Gamma_{1}\right)$. For each arrow $\boldsymbol{\rho}: \mathbf{i} \longrightarrow \mathbf{j}$ in $\Gamma$, define

$$
\varepsilon_{\boldsymbol{\rho}}=\#\{\text { arrows in } \boldsymbol{\rho}\}, d_{\boldsymbol{\rho}}=\varepsilon_{\boldsymbol{\rho}} / \varepsilon_{\mathbf{j}}, \text { and } d_{\boldsymbol{\rho}}^{\prime}=\varepsilon_{\boldsymbol{\rho}} / \varepsilon_{\mathbf{i}},
$$

where $\varepsilon_{\mathbf{k}}=\#\{$ vertices in the $\sigma$-orbit $\mathbf{k}\}$ for $\mathbf{k} \in I$. The quiver $\Gamma$ together with the valuation $\left\{\varepsilon_{\mathbf{d}}\right\}_{\mathbf{d} \in \Gamma_{0}},\left\{\left(d_{\boldsymbol{\rho}}, d_{\boldsymbol{\rho}}^{\prime}\right)\right\}_{\boldsymbol{\rho} \in \Gamma_{1}}$ defines a valued quiver $\Gamma=\Gamma(Q, \sigma)$. Clearly, each valued quiver can be obtained in this way from an ad-quiver.

Using the Frobenius morphism $F=F_{Q, \sigma}$ on $A$ defined in (3.5.1), we can attach

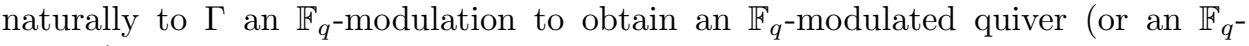
species) as follows: for each vertex $\mathbf{i} \in I$ and each arrow $\boldsymbol{\rho}$ in $\Gamma$, we fix $i_{0} \in \mathbf{i}$, $\rho_{0} \in \boldsymbol{\rho}$, and consider the $F_{A}$-stable subspaces of $A$

$$
A_{\mathbf{i}}=\bigoplus_{i \in \mathbf{i}} k e_{i}=\bigoplus_{s=0}^{\varepsilon_{\mathbf{i}}-1} k e_{\sigma^{s}\left(i_{0}\right)} \text { and } A_{\boldsymbol{\rho}}=\bigoplus_{\rho \in \rho} k \rho=\bigoplus_{t=0}^{\varepsilon_{\rho}-1} k \sigma^{t}\left(\rho_{0}\right)
$$

where $e_{i}$ denotes the idempotent corresponding to the vertex $i$. Then

$$
A_{\mathbf{i}}^{F}=\left\{\sum_{s=0}^{\varepsilon_{\mathbf{i}}-1} x^{q^{s}} e_{\sigma^{s}\left(i_{0}\right)} \mid x \in k, x^{q^{\varepsilon_{\mathbf{i}}}}=x\right\} \text { and } A_{\boldsymbol{\rho}}^{F}=\left\{\sum_{t=0}^{\varepsilon_{\boldsymbol{\rho}}-1} x^{q^{t}} \sigma^{t}\left(\rho_{0}\right) \mid x \in k, x^{q^{\varepsilon_{\boldsymbol{\rho}}}}=x\right\} \text {. }
$$

Further, the algebra structure of $A$ induces an $A_{\mathbf{j}}^{F}$ - $A_{\mathbf{i}}^{F}$-bimodule structure on $A_{\boldsymbol{\rho}}^{F}$ for each arrow $\boldsymbol{\rho}: \mathbf{i} \longrightarrow \mathbf{j}$ in $\Gamma$. Thus, we obtain an $\mathbb{F}_{q}$-modulation $\mathbb{M}=\mathbb{M}(Q, \sigma):=$ $\left(\left\{A_{\mathbf{i}}^{F}\right\}_{\mathbf{i}},\left\{A_{\boldsymbol{\rho}}^{F}\right\}_{\boldsymbol{\rho}}\right)$ over the valued quiver $\Gamma$. We shall denote the $\mathbb{F}_{q}$-modulated quiver defined above by

$$
\mathfrak{M}_{Q, \sigma}=\mathfrak{M}_{Q, \sigma ; q}=(\Gamma, \mathbb{M}) .
$$

Let $T\left(\mathfrak{M}_{Q, \sigma}\right)$ be the tensor algebra of the modulated quiver $\mathfrak{M}_{Q, \sigma}$. Thus, by definition, $T\left(\mathfrak{M}_{Q, \sigma}\right)=\bigoplus_{n \geqslant 0} M^{\otimes n}$, where $M=\bigoplus_{\rho \in \Gamma_{1}} A_{\rho}^{F}$ is viewed as an $R$ - $R$ bimodule with $R=\bigoplus_{\mathbf{i} \in I} A_{\mathbf{i}}^{F}$ and $\bigotimes=\bigotimes_{R}$. If, for each $\sigma$-orbit $\mathbf{p}$ of a path $\rho_{n} \cdots \rho_{2} \rho_{1}$ in $Q$, we set $A_{\mathbf{p}}=\bigoplus_{p \in \mathbf{p}} k p$. Then

$$
A_{\mathbf{p}}^{F} \cong A_{\boldsymbol{\rho}_{n}}^{F} \otimes_{\mathbb{F}_{n-1}} \cdots \otimes_{\mathbb{F}_{2}} A_{\boldsymbol{\rho}_{2}}^{F} \otimes_{\mathbb{F}_{1}} A_{\boldsymbol{\rho}_{1}}^{F},
$$

where $\boldsymbol{\rho}_{t}$ is the $\sigma$-orbit of $\rho_{t}$ and $\mathbb{F}_{t}=A_{h \boldsymbol{\rho}_{t}}^{F}$. Since $A^{F}=\bigoplus_{\mathbf{p}} A_{\mathbf{p}}^{F}$, it follows that the fixed point algebra $A^{F}$ is isomorphic to the tensor algebra $T\left(\mathfrak{M}_{Q, \sigma}\right)$. Thus, 
$A^{F}$-modules can be identified with representations of the modulated quiver $\mathfrak{M}_{Q, \sigma}$ (see [10]). The above observation together with Theorem 3.2 implies the following.

Proposition 6.3. Let $(Q, \sigma)$ be an ad-quiver with path algebra $A=k Q$ and induced Frobenius morphism $F=F_{Q, \sigma}$. Let $\mathfrak{M}_{Q, \sigma}$ be the associated $\mathbb{F}_{q}$-modulated quiver defined as above.

(a) We have an algebra isomorphism $A^{F} \cong T\left(\mathfrak{M}_{Q, \sigma}\right)$. Hence the categories $\bmod ^{F}-A$ and $\bmod -T\left(\mathfrak{M}_{Q, \sigma}\right)$ are equivalent.

(b) If $Q$ has no oriented cycles, then the fixed point algebra $A^{F}$ is a finitedimensional hereditary basic algebra.

Corollary 6.4. Maintain the notation above and let $r \geqslant 1$ be an integer. Then, the ad-quiver $\left(Q, \sigma^{r}\right)$ defines an $\mathbb{F}_{q^{r} \text {-modulated quiver }} \mathfrak{M}_{Q, \sigma^{r} ; q^{r}}$, whose tensor algebra

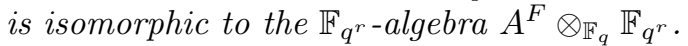

Proof. Clearly, $F_{Q, \sigma^{r} ; q^{r}}=F_{Q, \sigma ; q}^{r}=F^{r}$. By Lemma 2.1. we have $A=A^{F} \otimes_{\mathbb{F}_{q}} \overline{\mathbb{F}}_{q}$ and $F(a \otimes \lambda)=a \otimes \lambda^{q}$. Then $A^{F^{r}}=A^{F} \otimes_{\mathbb{F}_{q}} \mathbb{F}_{q^{r}}$. Now, the isomorphism follows from Proposition 6.3(a).

Note that for $r>1$, the modulated quiver $\mathfrak{M}_{Q, \sigma^{r} ; q^{r}}$ is different from the modulated quivers $\mathfrak{M}_{Q, \sigma^{r} ; q}$ and $\mathfrak{M}_{Q, \sigma ; q^{r}}$. The former has the same underlying valued quiver as $\mathfrak{M}_{Q, \sigma^{r} ; q^{r}}$, but different base field, while the latter has the same base field but different underlying valued quiver (if $\sigma \neq 1$ ).

Our next result shows that the converse of Proposition 6.3(b) is also true.

Theorem 6.5. Let $B$ be a finite-dimensional hereditary basic algebra over $\mathbb{F}_{q}$. Then there is an ad-quiver $(Q, \sigma)$ such that $B$ is isomorphic to $(k Q)^{F_{Q, \sigma}}$.

Proof. Let $A=B \otimes k$ and define $F: A \rightarrow A$ by $F(b \otimes \lambda)=b \otimes \lambda^{q}$. Clearly, $F$ is a Frobenius morphism on the $k$-algebra $A$ and $A^{F}=B$. Since $B$ is a finitedimensional hereditary basic algebra, it follows that so is $A$, and $B$ is isomorphic to the tensor (or path) algebra of the associated modulated Ext-quiver $Q_{B}$ (see [13, Theorem 8.5.2] or [2, p. 104]). In particular, we have an algebra isomorphism

$$
\varphi: B \stackrel{\sim}{\longrightarrow} \operatorname{gr} B, \quad \text { where } \quad \operatorname{gr} B=\bigoplus_{i \geqslant 0} \operatorname{Rad}^{i} B / \operatorname{Rad}^{i+1} B .
$$

Since $\operatorname{Rad} A=\operatorname{Rad}(B \otimes k)=(\operatorname{Rad} B) \otimes k$ (see, e.g., [5, p. 146]), we have by induction $\operatorname{Rad}^{i} A=\left(\operatorname{Rad}^{i} B\right) \otimes k$ for all $i \geqslant 1$. Thus, the isomorphism $\varphi$ induces a $k$-algebra isomorphism

$$
\tilde{\varphi}: A=B \otimes k \stackrel{\sim}{\longrightarrow} \operatorname{gr} A=\operatorname{gr} B \otimes k .
$$

Let $\bar{F}$ denote the Frobenius morphism on gr $A$ induced from $F$. Clearly, $\bar{F}$ stabilizes each direct summand $\operatorname{Rad}^{i} A / \operatorname{Rad}^{i+1} A$ of gr $A$ and $B=A^{F} \cong(\operatorname{gr} A)^{\bar{F}}$.

We now prove that $(\operatorname{gr} A, \bar{F})$ defines an ad-quiver $(Q, \sigma)$ such that $(\operatorname{gr} A)^{\bar{F}} \cong$ $(k Q)^{F_{Q, \sigma}}$. Suppose

$$
A / \operatorname{Rad} A=k \bar{e}_{1} \oplus \cdots \oplus k \bar{e}_{n},
$$

where $e_{i}$ are primitive orthogonal idempotents of $A$ with $1=e_{1}+\cdots+e_{n}$. Since the $F\left(e_{i}\right)$ 's form a complete set of primitive orthogonal idempotents of $A$, it follows that there is a permutation $\sigma$ and an invertible element $u \in A$ such that $F\left(e_{i}\right)=u e_{\sigma i} u^{-1}$. Thus, $\bar{F}\left(\bar{e}_{i}\right)=\bar{e}_{\sigma i}$ for all $i$. 
Let $Q_{0}=\{1,2, \ldots, n\}$ and $I$ the set of $\sigma$-orbits. Putting $f_{\mathbf{i}}=\sum_{i \in \mathbf{i}} e_{i}$ for each $\mathbf{i} \in I$, we have

$$
\operatorname{Rad} A / \operatorname{Rad}^{2} A=\bigoplus_{\mathbf{i}, \mathbf{j} \in I} f_{\mathbf{i}}\left(\operatorname{Rad} A / \operatorname{Rad}^{2} A\right) f_{\mathbf{j}}
$$

where

$$
f_{\mathbf{i}}\left(\operatorname{Rad} A / \operatorname{Rad}^{2} A\right) f_{\mathbf{j}}=\bar{f}_{\mathbf{i}}\left(\operatorname{Rad} A / \operatorname{Rad}^{2} A\right) \bar{f}_{\mathbf{j}}=\bigoplus_{i \in \mathbf{i}, j \in \mathbf{j}} \bar{e}_{i}\left(\operatorname{Rad} A / \operatorname{Rad}^{2} A\right) \bar{e}_{j} .
$$

Clearly, $\bar{F}$ stabilizes each summand $f_{\mathbf{i}}\left(\operatorname{Rad} A / \operatorname{Rad}^{2} A\right) f_{\mathbf{j}}$ and $\bar{F}\left(V_{i j}\right)=V_{\sigma i, \sigma j}$, where

$$
V_{i j}=\bar{e}_{i}\left(\operatorname{Rad} A / \operatorname{Rad}^{2} A\right) \bar{e}_{j}
$$

Fix $i, j$ and let $s$ be the smallest integer such that $\sigma^{s} i=i$ and $\sigma^{s} j=j$. Since $\bar{F}^{s}$ stabilizes $V_{i j}$, we can choose a $k$-basis $v_{1}, \ldots, v_{t_{i j}}$ of $V_{i j}$ such that $\bar{F}^{s}\left(v_{a}\right)=v_{a}$ for all $1 \leqslant a \leqslant t_{i j}$. Thus, we obtain a $k$-basis

$$
\left\{v_{1}, \ldots, v_{t_{i j}}, \bar{F}\left(v_{1}\right), \ldots, \bar{F}\left(v_{t_{i j}}\right), \ldots, \bar{F}^{s-1}\left(v_{1}\right), \ldots, \bar{F}^{s-1}\left(v_{t_{i j}}\right)\right\}
$$

for the $\bar{F}$-stable space $\bigoplus_{a=0}^{s-1} V_{\sigma^{a} i, \sigma^{a} j}$. Clearly, such a basis can be constructed for every $\sigma$-orbit of the set $\{(i, j) \mid i \in \mathbf{i}, j \in \mathbf{j}\}$. Thus, we obtain a basis $\left\{v_{a}^{i j}\right\}_{i, j, a}$ for $f_{\mathbf{i}}\left(\operatorname{Rad} A / \operatorname{Rad}^{2} A\right) f_{\mathbf{j}}$, and hence for $\operatorname{Rad} A / \operatorname{Rad}^{2} A$, which is $\bar{F}$-stable, i.e., $\left\{\bar{F}\left(v_{a}^{i j}\right)\right\}_{i, j, a}=\left\{v_{a}^{i j}\right\}_{i, j, a}$. If $Q_{1}$ is the set of arrows $\rho_{a}^{i j}: i \rightarrow j$ indexed by the basis elements $v_{a}^{i j}$, then $\bar{F}$ induces a permutation $\sigma$ on $Q_{1}$.

So we have obtained an ad-quiver $(Q, \sigma)$. The standard $k$-algebra isomorphism $\psi: k Q \rightarrow \operatorname{gr} A$ sending $i$ to $\bar{e}_{i}$ and $\rho_{a}^{i j}$ to $v_{a}^{i j}$ is compatible with the Frobenius morphisms $\bar{F}$ and $F_{Q, \sigma}$, that is, $\psi \circ F_{Q, \sigma}=\bar{F} \circ \psi$. Consequently, we obtain

$$
B \cong(\operatorname{gr} A)^{\bar{F}} \cong(k Q)^{F_{Q, \sigma}} .
$$

Corollary 6.6. Every finite-dimensional hereditary algebra over a finite field is Morita equivalent to the F-fixed point algebra of the path algebra of an ad-quiver.

Remarks 6.7. (1) If we identify $A_{\mathbf{i}}^{F}$ and $A_{\boldsymbol{\rho}}^{F}$ defined in (6.2.2) with $\mathbb{F}_{q^{\varepsilon_{\mathbf{i}}}}$ and $\mathbb{F}_{q^{\varepsilon} \boldsymbol{\rho}}$ via

$$
\sum_{i \in \mathbf{i}} x_{i} e_{i} \longmapsto x_{i_{0}} \text { and } \sum_{\rho \in \boldsymbol{\rho}} y_{\rho} \rho \longmapsto y_{\rho_{0}},
$$

respectively, where $i_{0} \in \mathbf{i}$ and $j_{0} \in \mathbf{j}$ are fixed, then, for an arrow $\boldsymbol{\rho}: \mathbf{i} \rightarrow \mathbf{j}$, the

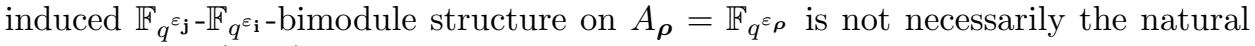
bimodule $\mathbb{F}_{q} \varepsilon_{\mathbf{j}}\left(\mathbb{F}_{q^{\varepsilon} \boldsymbol{\rho}}\right)_{\mathbb{F}_{q} \varepsilon_{\mathbf{i}}}$ induced by the subfield structure. This is because, for $r, s \geqslant 1$, the $\mathbb{F}_{q}$-algebra isomorphism

$$
\mathbb{F}_{q^{r}} \otimes_{\mathbb{F}_{q}} \mathbb{F}_{q^{s}} \cong \underbrace{\mathbb{F}_{q^{m}} \times \cdots \times \mathbb{F}_{q^{m}}}_{d}
$$

means that there are exactly $d$ non-isomorphic simple $\mathbb{F}_{q^{r}}-\mathbb{F}_{q^{s}}$-bimodules. Here $d$ and $m$ denote the greatest common divisor and the least common multiple of $r, s$, respectively. More precisely, for $0 \leqslant r \leqslant \varepsilon_{\mathbf{i}}-1$ and $0 \leqslant s \leqslant \varepsilon_{\mathbf{j}}-1$ with

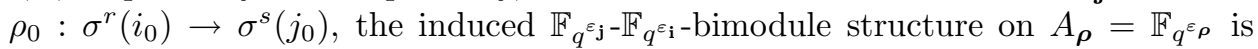
given by

$$
y \cdot z \cdot x=y^{q^{s}} z x^{q^{r}} \text { for } x \in \mathbb{F}_{q^{\varepsilon_{\mathbf{i}}}}, y \in \mathbb{F}_{q^{\varepsilon_{\mathbf{j}}}}, z \in \mathbb{F}_{q^{\varepsilon_{\boldsymbol{\rho}}}},
$$


while the natural bimodule structure corresponds to the case when $r=s=0$. These two bimodule structures on $\mathbb{F}_{q^{\varepsilon} \rho}$ are not necessarily isomorphic.

(2) The $\mathbb{F}_{q}$-modulated quivers studied in [19] and 21] involve only the natural bimodules $\mathbb{F}_{q^{r}}\left(\mathbb{F}_{q^{n}}\right)_{\mathbb{F}_{q^{s}}}$, where $r, s, n \geqslant 1, r \mid n$ and $s \mid n$. These will be called natural modulated quivers below. Note that the natural $\mathbb{F}_{q^{r}}-\mathbb{F}_{q^{s}}$-bimodule $\mathbb{F}_{q^{r}}\left(\mathbb{F}_{q^{n}}\right)_{\mathbb{F}_{q^{s}}}$ is isomorphic to the direct sum of $\frac{n}{m}$ copies of the natural simple bimodule $\mathbb{F}_{q^{r}}\left(\mathbb{F}_{q^{m}}\right)_{\mathbb{F}_{q^{s}}}$, where $m$ is the least common multiple of $r$ and $s$. Though non-isomorphic bimodules involved in two modulated quivers could result in isomorphic tensor algebras, the following example shows that not every finite dimensional hereditary basic algebra over $\mathbb{F}_{q}$ arises from a natural modulated quiver.

Example 6.8. Let $\Gamma$ denote the valued quiver



with $\left(\varepsilon_{a}, \varepsilon_{b}, \varepsilon_{c}\right)=(1,2,2),\left(d_{\rho}, d_{\rho}^{\prime}\right)=(1,2)$, and $\left(d_{\tau}, d_{\tau}^{\prime}\right)=(2,2)$. Then the pair of natural bimodules $\mathbb{F}_{q^{2}}\left(\mathbb{F}_{q^{2}}\right)_{\mathbb{F}_{q}}$ and $\mathbb{F}_{q^{2}}\left(\mathbb{F}_{q^{4}}\right)_{\mathbb{F}_{q^{2}}}$, together with the valued quiver $\Gamma$, defines the natural $\mathbb{F}_{q}$-modulated quiver $\mathfrak{M}$. Let $M$ denote the natural bimodule $\mathbb{F}_{q}^{2}\left(\mathbb{F}_{q^{2}}\right)_{\mathbb{F}_{q^{2}}}$ and let $M^{\prime}=\mathbb{F}_{q^{2}}$ (as $\mathbb{F}_{q^{\text {-vector }}}$ spaces) denote the $\mathbb{F}_{q^{2}}-\mathbb{F}_{q^{2}}$-bimodule given by $x \cdot y \cdot z=x^{q} y z$. It is easy to see that $M$ and $M^{\prime}$ are not isomorphic as $\mathbb{F}_{q^{2}}-\mathbb{F}_{q^{2}}$-bimodules. The pair of bimodules $\mathbb{F}_{q^{2}}\left(\mathbb{F}_{q^{2}}\right)_{\mathbb{F}_{q}}$ and $M \oplus M^{\prime}$ also defines a new $\mathbb{F}_{q}$-modulated quiver $\mathfrak{M}^{\prime}$ whose tensor algebra $T\left(\mathfrak{M}^{\prime}\right)$ is not isomorphic to the tensor algebra $T(\mathfrak{M})$ of $\mathfrak{M}$, since $T(\mathfrak{M}) \otimes_{\mathbb{F} q} k$ and $T\left(\mathfrak{M}^{\prime}\right) \otimes_{\mathbb{F} q} k$ are respectively isomorphic to the path algebras of the following quivers:
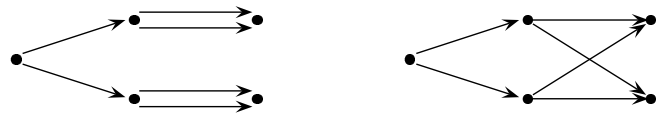

\section{Almost split sequences}

The Auslander-Reiten theory is one of the fundamental tools in the study of representations of algebras (see, e.g., 1 ). This and the next sections are devoted to establishing a relation between the Auslander-Reiten theories of $A$ and its fixed point algebra $A^{F}$.

We briefly review the general theory. Let $A$ be a finite-dimensional algebra over an arbitrary field $k$. A morphism $\varphi: L \rightarrow M$ in $\bmod -A$ is called minimal right almost split if

(a) $\varphi$ is not a split epimorphism,

(b) any morphism $X \rightarrow M$ which is not a split epimorphism factors through $\varphi$

(c) any morphism $f: L \rightarrow L$ satisfying $\varphi=\varphi f$ is an isomorphism.

It is easy to see that, if $\varphi: L \rightarrow M$ is a minimal right almost split morphism, then $M$ is indecomposable.

A minimal left almost split morphism is defined dually.

A short exact sequence $0 \rightarrow N \stackrel{\psi}{\rightarrow} L \stackrel{\varphi}{\rightarrow} M \rightarrow 0$ is called an almost split sequence if $\varphi$ is minimal right almost split, or equivalently, if $\psi$ is minimal left almost split. 
Theorem 7.1 (Auslander-Reiten). Let $M$ be an indecomposable A-module.

(a) There exists a unique, up to isomorphism, minimal right almost split morphism $\varphi: L \rightarrow M$. If, moreover, $M$ is not projective, then the sequence

$$
0 \longrightarrow \operatorname{Ker} \varphi \longrightarrow L \stackrel{\varphi}{\longrightarrow} M \longrightarrow 0
$$

is an almost split sequence.

(b) There exists a unique, up to isomorphism, minimal left almost split morphism $\psi: M \rightarrow L$. If, moreover, $M$ is not injective, then the sequence

$$
0 \longrightarrow M \stackrel{\psi}{\longrightarrow} L \longrightarrow \text { Coker } \psi \longrightarrow 0
$$

is an almost split sequence.

Given an almost split sequence $0 \rightarrow N \stackrel{\psi}{\rightarrow} L \stackrel{\varphi}{\rightarrow} M \rightarrow 0$, the module $N$ (resp. $M$ ) is uniquely (up to isomorphism) determined by $M$ (resp. $N$ ). We write $\tau_{A} M=N$ or $N=\tau_{A}^{-1} M$. The $\tau_{A}=: \tau$ is called the Auslander-Reiten translation of $A$, which indeed admits the following description using the transpose and the dual (see 1, Chap. IV, V]. Let $\mathcal{P}$ and $\mathcal{I}$ be the ideals of $\bmod -A$ (in the sense of [17, p. 16]) defined respectively by

$$
\mathcal{P}(X, Y)=\{f: X \rightarrow Y \mid f \text { factors through a projective } A \text {-module }\}
$$

and

$$
\mathcal{I}(X, Y)=\{f: X \rightarrow Y \mid f \text { factors through an injective } A \text {-module }\},
$$

where $X, Y \in \bmod -A$. The factor categories of $\bmod -A$ by $\mathcal{P}$ and $\mathcal{I}$ are denoted by $\underline{\bmod }-A$ and $\overline{\bmod }-A$, respectively. Let $M \in \bmod -A$ and let

$$
P_{1} \stackrel{f}{\longrightarrow} P_{0} \longrightarrow M \longrightarrow 0
$$

be a minimal projective presentation of $M$. Applying $\operatorname{Hom}_{A}(-, A)$, we get a morphism

$$
f^{*}:=\operatorname{Hom}_{A}(f, A): \operatorname{Hom}_{A}\left(P_{0}, A\right) \rightarrow \operatorname{Hom}_{A}\left(P_{1}, A\right)
$$

in mod- $A^{\mathrm{op}}$. Thus, we obtain an $A^{\mathrm{op}}$-module Coker $f^{*}$ which is called the transpose of $M$ and denoted by $\operatorname{Tr} M$. In general, $\operatorname{Tr}$ does not give rise to a functor from $\bmod -A$ to $\bmod -A^{\mathrm{op}}$. However, Tr induces a functor $\operatorname{Tr}: \underline{\bmod }-A \rightarrow \underline{\bmod }-A^{\mathrm{op}}$ such that the compositions $\underline{\text { mod}}-A \stackrel{\operatorname{Tr}}{\rightarrow} \underline{\text { mod}}-A$ op $\stackrel{\operatorname{Tr}}{\longrightarrow} \underline{\text { mod}}-A$ and $\underline{\text { mod}}-A \stackrel{\text { op }}{\longrightarrow} \underline{\operatorname{Tr}} \underline{\text { mod}}-A \stackrel{\operatorname{Tr}}{\longrightarrow}$ $\underline{\mathbf{m o d}}-A^{\mathrm{op}}$ are isomorphic to the identity on $\underline{\mathbf{m o d}}-A$ and $\underline{\mathbf{m o d}}-A^{\mathrm{op}}$, respectively. On the other hand, the duality $D=\operatorname{Hom}_{k}(-, k): \bmod -A \rightarrow \bmod -A^{\text {op }}$ induces a duality $D: \underline{\bmod }-A \rightarrow \overline{\bmod }-A^{\text {op }}$ such that the composition $D \operatorname{Tr}: \underline{\bmod }-A \rightarrow$ $\overline{\bmod }-A$ is an equivalence with inverse equivalence $\operatorname{Tr} D: \overline{\bmod }-A \rightarrow \underline{\bmod }-A$. Then, for each indecomposable non-projective $A$-module $M$, we have $\tau M \cong D \operatorname{Tr} M$, and for each indecomposable non-injective $A$-module $N$, we have $\tau^{-1} N \cong \operatorname{Tr} D N$.

For any two $A$-modules $M=\bigoplus_{i} M_{i}$ and $N=\bigoplus_{j} N_{j}$, where $M_{i}$ and $N_{j}$ are indecomposable, we define the radical of $\operatorname{Hom}_{A}(M, N)$ by

$\operatorname{Rad}_{A}(M, N)=\left\{\left(f_{j i}\right): M \rightarrow N \mid f_{j i}: M_{i} \rightarrow N_{j}\right.$ is not an isomorphism for all $\left.i, j\right\}$.

In fact, $\operatorname{Rad}_{A}(-,-)$ is an ideal of $\bmod -A$. Inductively, for each $n>1$, the $n$-th power of the radical is defined to be

$$
\operatorname{Rad}_{A}^{n}(M, N)=\sum_{X} \operatorname{Rad}_{A}^{n-1}(X, N) \circ \operatorname{Rad}_{A}(M, X) .
$$


From the functorial point of view (see, e.g., [16 and [2, 4.8]), we may study almost split sequences in the category $\operatorname{Fun}(A)$ (resp. Fun $\left.{ }^{\mathrm{op}}(A)\right)$ whose objects are the covariant (resp. contravariant) additive functors from mod- $A$ to the category mod- $k$ of finite-dimensional $k$-vector spaces, and whose morphisms are the natural transformations of functors. Thus, each $A$-module $M$ defines a functor $\operatorname{Hom}_{A}(-, M)$ in $\mathbf{F u n}^{\mathrm{op}}(A)$ by $\operatorname{Hom}_{A}(-, M)(N)=\operatorname{Hom}_{A}(N, M)$ and a subfunctor $\operatorname{Rad}_{A}(-, M)$ by $\operatorname{Rad}_{A}(-, M)(N)=\operatorname{Rad}_{A}(N, M)$. We denote the induced quotient functor by

$$
\mathcal{H}_{M}:=\operatorname{Hom}_{A}(-, M) / \operatorname{Rad}_{A}(-, M) \text {. }
$$

In particular, if $M$ is indecomposable, $\mathcal{H}_{M}$ is a simple functor - a functor that has no non-zero proper subfunctor. Dually, each $A$-module $M$ defines a functor $\operatorname{Hom}_{A}(M,-)$ in $\operatorname{Fun}(A)$ and the quotient functor $\mathcal{K}_{M}$ by its subfunctor $\operatorname{Rad}_{A}(M,-)$. We have the following functorial characterization of minimal right or left almost split morphisms; see [16, 1.4] or [2, 4.12.6].

Proposition 7.2. $\quad$ (a) A morphism $\varphi: L \rightarrow M$ of A-modules is minimal right almost split if and only if the induced sequence

$$
0 \longrightarrow \operatorname{Hom}_{A}(-, \operatorname{ker} \varphi) \stackrel{\kappa_{*}}{\longrightarrow} \operatorname{Hom}_{A}(-, L) \stackrel{\varphi_{*}}{\longrightarrow} \operatorname{Hom}_{A}(-, M) \stackrel{\xi_{M}}{\longrightarrow} \mathcal{H}_{M} \longrightarrow 0
$$

is a minimal projective resolution of $\mathcal{H}_{M}$ in $\mathbf{F u n}^{\mathrm{op}}(A)$, where $\kappa: \operatorname{Ker} \varphi \rightarrow L$ is the canonical inclusion and $\xi_{M}$ denotes the canonical projection.

(b) A morphism $\psi: N \rightarrow L$ of A-modules is minimal left almost split if and only if the induced sequence

$0 \longrightarrow \operatorname{Hom}_{A}($ Coker $\psi,-) \stackrel{\pi_{*}}{\longrightarrow} \operatorname{Hom}_{A}(L,-) \stackrel{\psi_{*}}{\longrightarrow} \operatorname{Hom}_{A}(N,-) \stackrel{\xi_{N}}{\longrightarrow} \mathcal{K}_{N} \longrightarrow 0$ is a minimal projective resolution of $\mathcal{K}_{N}$ in $\operatorname{Fun}(A)$, where $\pi: L \rightarrow$ Coker $\psi$ is the canonical projection.

We now assume $k=\overline{\mathbb{F}}_{q}$ and let $F_{A}$ be a fixed Frobenius morphism on $A$. We also assume that each $A$-module $M$ has an $\mathbb{F}_{q}$-structure given by a Frobenius map $F_{M}$.

Lemma 7.3. A morphism $\varphi: L \rightarrow M$ (resp. $\psi: N \rightarrow L$ ) in $\bmod -A$ is minimal right (resp. left) almost split if and only if $\varphi^{[1]}: L^{[1]} \rightarrow M^{[1]}$ (resp. $\psi^{[1]}: N^{[1]} \rightarrow$ $\left.L^{[1]}\right)$ is also. In particular, a sequence $0 \rightarrow N \stackrel{\psi}{\rightarrow} L \stackrel{\varphi}{\rightarrow} M \rightarrow 0$ is an almost split sequence if and only if so is $0 \rightarrow N^{[1]} \stackrel{\psi^{[1]}}{\rightarrow} L^{[1]} \stackrel{\varphi^{[1]}}{\rightarrow} M^{[1]} \rightarrow 0$. Moreover, $\tau M^{[1]} \cong(\tau M)^{[1]}$.

Proof. Since the Frobenius twisting functor ()$^{[1]}$ is invertible with the inverse ()$^{[-1]}$ given in (4.1.1), everything is clear.

Let $\varphi: L \rightarrow M$ be a minimal right almost split morphism in mod- $A$. Then, by the lemma above, for each integer $s \geqslant 1, \varphi^{[s]}: L^{[s]} \rightarrow M^{[s]}$ is minimal right almost split. Let $r=p(M)$ be the $F$-period of $M$, i.e., $r$ is minimal with $M^{[r]} \cong M$. Then Theorem 7.1 implies $L^{[r]} \cong L$. By Remarks 4.5(2), we may assume that $M^{[r]}=M$ and $L^{[r]}=L$ as $A$-modules. Thus, both $\tilde{M}=\bigoplus_{i=0}^{r-1} M^{[i]}$ and $\tilde{L}=\bigoplus_{i=0}^{r-1} L^{[i]}$ defined in (5.0.1) are $F$-stable with respect to Frobenius maps $F_{\tilde{M}}$ and $F_{\tilde{L}}$ defined by $F_{M}$ and $F_{L}$, respectively (see (5.0.2) $)$. Since both $\varphi: L \rightarrow M$ and $\varphi^{[r]}: L \rightarrow M$ are minimal right almost split, there is a $g \in \operatorname{Aut}_{A}(L)$ such that $\varphi=\varphi^{[r]} \circ g$. Let $F=F_{(L, L)}$ and $F_{(L, M)}$ be the induced Frobenius maps on $\operatorname{Hom}_{A}(L, L)$ and 
$\operatorname{Hom}_{A}(L, M)$, respectively; see Lemma 2.3. Then $F_{(L, M)}(\psi \circ f)=F_{(L, M)}(\psi) \circ F(f)$ for all $\psi \in \operatorname{Hom}_{A}(L, M)$ and $f \in \operatorname{End}_{A}(L)$. Restricting $F$ to the connected algebraic group $\operatorname{Aut}_{A}(L)$ and applying Lang-Steinberg's theorem, we may find an element $h \in \operatorname{Aut}_{A}(L)$ satisfying $g=F^{r}(h) h^{-1}$, that is, $h=g^{-1} F^{r}(h)$. This implies that

$$
\varphi \circ h=\varphi \circ\left(g^{-1} F^{r}(h)\right)=\varphi^{[r]} \circ F^{r}(h)=F_{(L, M)}^{r}(\varphi \circ h) .
$$

Note that, by Proposition 4.2. $F_{(L, M)}^{r}(\varphi)=\varphi^{[r]}$. Thus, $\varphi \circ h: L \rightarrow M$ is minimal right almost split satisfying $(\varphi \circ h)^{[r]}=\varphi \circ h$. Replacing $\varphi$ by $\varphi \circ h$, we may assume that $\varphi$ is chosen to satisfy $\varphi^{[r]}=\varphi$. Now we define

$$
\tilde{\varphi}:=\operatorname{diag}\left(\varphi, \varphi^{[1]}, \ldots, \varphi^{[r-1]}\right): \tilde{L}=\bigoplus_{i=0}^{r-1} L^{[i]} \rightarrow \bigoplus_{i=0}^{r-1} M^{[i]}=\tilde{M} .
$$

The equality $\varphi^{[r]}=\varphi$ implies that $\tilde{\varphi}$ is a morphism in $\bmod ^{F}-A$. Hence, $\tilde{\varphi}$ induces an $A^{F}$-module morphism $\tilde{\varphi}^{F}: \tilde{L}^{F} \rightarrow \tilde{M}^{F}$. Here again we drop the subscripts of the $F$ 's for notational simplicity.

Theorem 7.4. Let $\varphi: L \rightarrow M$ be a minimal right almost split morphism. Then there exists an induced morphism $\tilde{\varphi}: \tilde{L} \rightarrow \tilde{M}$ of F-stable modules such that its restriction $\tilde{\varphi}^{F}: \tilde{L}^{F} \rightarrow \tilde{M}^{F}$ is a minimal right almost split morphism in $\bmod -A^{F}$. In particular, every almost split sequence $0 \rightarrow N \stackrel{\psi}{\rightarrow} L \stackrel{\varphi}{\rightarrow} M \rightarrow 0$ in $\bmod -A$ gives rise to an almost split sequence

$$
0 \longrightarrow \tilde{N}^{F} \stackrel{\tilde{\psi}^{F}}{\longrightarrow} \tilde{L}^{F} \stackrel{\tilde{\varphi}^{F}}{\longrightarrow} \tilde{M}^{F} \longrightarrow 0
$$

in $\bmod -A^{F}$. Moreover, every almost split sequence of $A^{F}$-modules can be constructed in this way.

Proof. Clearly, the last assertion follows from Theorem 5.1. From the construction of $\tilde{\varphi}$ above, we have $\operatorname{Ker} \tilde{\varphi}=\bigoplus_{i=0}^{r-1} \operatorname{Ker} \varphi^{[i]}$, and the Frobenius map $F_{\tilde{L}}$ on $\tilde{L}$ induces a Frobenius map on $\operatorname{Ker} \tilde{\varphi}$. Thus, $\operatorname{Ker} \tilde{\varphi}$ is $F$-stable.

For each $0 \leqslant i \leqslant r-1$, by Proposition 7.2 and Lemma 7.3, the morphism $\varphi^{[i]}: L^{[i]} \rightarrow M^{[i]}$ gives the minimal projective resolution of $\mathcal{H}_{M^{[i]}}$

$$
0 \longrightarrow \operatorname{Hom}_{A}\left(-, \operatorname{Ker} \varphi^{[i]}\right) \stackrel{\kappa_{*}^{[i]}}{\longrightarrow} \operatorname{Hom}_{A}\left(-, L^{[i]}\right) \stackrel{\varphi_{*}^{[i]}}{\longrightarrow} \operatorname{Hom}_{A}\left(-, M^{[i]}\right) \stackrel{\xi_{M^{[i]}}}{\longrightarrow} \mathcal{H}_{M^{[i]}} \longrightarrow 0
$$

where $\kappa^{[i]}$ is the inclusion $\operatorname{Ker} \varphi^{[i]} \rightarrow L^{[i]}$. Summing up, we obtain a minimal projective resolution of $\mathcal{H}_{\tilde{M}} \cong \bigoplus_{i=0}^{r-1} \mathcal{H}_{M^{[i]}}$

$$
0 \longrightarrow \operatorname{Hom}_{A}(-, \operatorname{Ker} \tilde{\varphi}) \stackrel{\tilde{\kappa}_{*}}{\longrightarrow} \operatorname{Hom}_{A}(-, \tilde{L}) \stackrel{\tilde{\varphi}_{*}}{\longrightarrow} \operatorname{Hom}_{A}(-, \tilde{M}) \stackrel{\xi_{\tilde{M}}}{\longrightarrow} \mathcal{H}_{\tilde{M}} \longrightarrow 0,
$$

where $\tilde{\kappa}=\operatorname{diag}\left\{\kappa^{[i]}\right\}: \operatorname{Ker} \tilde{\varphi}=\bigoplus_{i=0}^{r-1} \operatorname{Ker} \varphi^{[i]} \rightarrow \tilde{L}$. Thus, for each $F$-stable module $\left(X, F_{X}\right)$, we get the following exact sequence:

$$
0 \longrightarrow \operatorname{Hom}_{A}(X, \operatorname{Ker} \tilde{\varphi}) \stackrel{\tilde{\kappa}_{*}(X)}{\longrightarrow} \operatorname{Hom}_{A}(X, \tilde{L}) \stackrel{\tilde{\varphi}_{*}(X)}{\longrightarrow} \operatorname{Hom}_{A}(X, \tilde{M}) \stackrel{\xi_{\tilde{I}}(X)}{\longrightarrow} \mathcal{H}_{\tilde{M}}(X) \longrightarrow 0 .
$$

Now the Frobenius maps on modules induce Frobenius maps $\tilde{F}$ on each space in the sequence above. It is easy to see that all morphisms in the sequence are compatible with those Frobenius maps $\tilde{F}$. This gives the exact sequence

$$
0 \longrightarrow \operatorname{Hom}_{A}(X, \operatorname{Ker} \tilde{\varphi})^{\tilde{F}} \longrightarrow \operatorname{Hom}_{A}(X, \tilde{L})^{\tilde{F}} \longrightarrow \operatorname{Hom}_{A}(X, \tilde{M})^{\tilde{F}} \longrightarrow \mathcal{H}_{\tilde{M}}(X)^{\tilde{F}} \longrightarrow 0
$$


Then we deduce from Proposition 3.4 the following exact sequence:

$$
\begin{aligned}
0 & \rightarrow \operatorname{Hom}_{A^{F}}\left(X^{F}, \operatorname{Ker}\left(\tilde{\varphi}^{F}\right)\right) \rightarrow \operatorname{Hom}_{A^{F}}\left(X^{F}, \tilde{L}^{F}\right) \\
& \rightarrow \operatorname{Hom}_{A^{F}}\left(X^{F}, \tilde{M}^{F}\right) \rightarrow \mathcal{H}_{\tilde{M}^{F}}\left(X^{F}\right) \rightarrow 0 .
\end{aligned}
$$

Since every $A^{F}$-module is of the form $X^{F}$ (Theorem [3.2), we obtain a minimal projective resolution of the simple functor $\mathcal{H}_{\tilde{M}^{F}}$ in $\operatorname{Fun}\left(A^{F}\right)$

$$
\begin{aligned}
& 0 \longrightarrow \operatorname{Hom}_{A^{F}}\left(-, \operatorname{Ker}\left(\tilde{\varphi}^{F}\right)\right) \stackrel{\tilde{\kappa}_{*}^{F}}{\longrightarrow} \operatorname{Hom}_{A^{F}}\left(-, \tilde{L}^{F}\right) \\
& \stackrel{\tilde{\varphi}_{*}^{F}}{\longrightarrow} \operatorname{Hom}_{A^{F}}\left(-, \tilde{M}^{F}\right) \stackrel{\xi_{\tilde{M}^{F}}}{\longrightarrow} \mathcal{H}_{\tilde{M}^{F}} \longrightarrow 0 .
\end{aligned}
$$

Therefore, by Proposition 7.2 again, $\tilde{\varphi}^{F}: \tilde{L}^{F} \rightarrow \tilde{M}^{F}$ is a minimal right almost split morphism in mod- $A^{F}$.

Dually, for each minimal left almost split morphism $\psi: N \rightarrow L$ in $\bmod -A$, we can construct a minimal left almost split morphism $\tilde{\psi}^{F}: \tilde{N}^{F} \rightarrow \tilde{L}^{F}$ in $\bmod -A^{F}$ in a similar way. We leave the details to the reader.

\section{Auslander-Reiten quivers}

We are now going to prove that the Frobenius morphism $F$ on $A$ induces an automorphism $\mathfrak{s}$ of the Auslander-Reiten quiver $\mathcal{Q}$ of $A$ and that the induced modulated quiver $\mathfrak{M}_{\mathcal{Q}, \mathfrak{s}}$ is isomorphic to the Auslander-Reiten quiver of $A^{F}$.

We begin with the general definition. Let $A$ be a finite-dimensional algebra over an arbitrary field $k$. For an $A$-module $M$, let $D_{M}$ denote the $k$-algebra

$$
D_{M}:=\operatorname{End}_{A}(M) / \operatorname{Rad}\left(\operatorname{End}_{A}(M)\right) .
$$

This is a division algebra if $M$ is indecomposable. By definition, the AuslanderReiten quiver (or $A R$-quiver for short) of $A$ is a (simple) $k$-modulated quiver $\mathcal{Q}_{A}$ consisting of a valued graph $\Gamma=\Gamma_{A}$ and a $k$-modulation $\mathbb{M}=\mathbb{M}_{A}$ defined on $\Gamma$. Here, the vertices of $\Gamma$ are isoclasses $[M]$ of indecomposable $A$-modules and the arrows $[M] \rightarrow[N]$ for indecomposable $M$ and $N$ are defined by the condition $\operatorname{Irr}_{A}(M, N) \neq 0$, where

$$
\operatorname{Irr}_{A}(M, N):=\operatorname{Rad}_{A}(M, N) / \operatorname{Rad}_{A}^{2}(M, N)
$$

is the space of irreducible homomorphisms from $M$ to $N$. Each arrow $[M] \rightarrow[N]$ has the valuation $\left(d_{M N}, d_{M N}^{\prime}\right)$, with $d_{M N}$ and $d_{M N}^{\prime}$ being the dimensions of $\operatorname{Irr}_{A}(M, N)$ considered as left $D_{N}$-space and right $D_{M}$-space, respectively. The $k$-modulation $\mathbb{M}$ is given by division algebras $D_{M}$ for vertices $[M]$ and (non-zero) $D_{N}-D_{M}$-bimodules $\operatorname{Irr}_{A}(M, N)$ for arrows $[M] \rightarrow[N]$.

Remarks 8.1. (1) The AR-quiver of $A$ defined in [1, VII.1] is simply the valued quiver $\Gamma_{A}$ together with the translation $\tau$ sending non-projective vertices to noninjective vertices. The modulation $\mathbb{M}_{A}$ for $\mathcal{Q}_{A}$ is not explicitly mentioned there. Here we adopt the definition for AR-quivers given by Benson in [2, p. 150]. We will see that this definition is more natural to fit our situation.

(2) The valuation $\left(d, d^{\prime}\right)$ of an arrow $[M] \rightarrow[N]$ in $\mathcal{Q}_{A}$ admits the following description (see [1, VII.1]). If $L \rightarrow M$ is minimal right almost split, then $L \cong$ $d^{\prime} M \oplus L_{1}$, where $L_{1}$ has no summand isomorphic to $M$. If $N \rightarrow K$ is minimal left almost split, then $K \cong d N \oplus K_{1}$, where $K_{1}$ admits no summand isomorphic to $N$. 
(3) If $k$ is algebraically closed, then $D_{M} \cong k$ and $d_{M N}=d_{M N}^{\prime}=\operatorname{dim}_{k} \operatorname{Irr}(M, N)$ for all indecomposable $A$-modules $M$ and $N$. So the modulation in the AR-quiver $\mathcal{Q}_{A}$ consists of $k$-spaces which can be represented by drawing $d_{M N}$ arrows from $[M]$ to $[N]$. In this way, we turn the modulated quiver $\mathcal{Q}_{A}$ to an ordinary quiver.

We return to our usual setup: let $k=\overline{\mathbb{F}}_{q}$ and let $F_{A}$ be a fixed Frobenius morphism on $A$. Given an indecomposable $A$-module $M$, let $r=p(M)$ be the $F$-period of $M$. In view of Remarks 4.5)(2), we may assume that $M^{[r]}=M$ (as $A$-modules). Then $\tilde{M}=M \oplus M^{[1]} \oplus \cdots \oplus M^{[r-1]}$ is $F$-stable with $F_{\tilde{M}}: \tilde{M} \rightarrow \tilde{M}$ defined by

$$
F_{\tilde{M}}\left(x_{0}, x_{1}, \ldots, x_{r-1}\right)=\left(F_{M}\left(x_{r-1}\right), F_{M}\left(x_{0}\right), \ldots, F_{M}\left(x_{r-2}\right)\right) .
$$

By Theorem 5.1, $\tilde{M}^{F}$ is an indecomposable $A^{F}$-module. By Proposition 3.4 the induced Frobenius map $F=F_{(\tilde{M}, \tilde{M})}$ on $\operatorname{End}_{A}(\tilde{M})$ gives a canonical $\mathbb{F}_{q}$-algebra isomorphism



In particular, if $\tilde{M}=X_{k}:=X \otimes k$ for an indecomposable $A^{F}$-module $X$, then

$$
\left(\operatorname{End}_{A}(\tilde{M}) / \operatorname{Rad} \operatorname{End}_{A}(\tilde{M})\right)^{F}=\left(D_{X_{k}}\right)^{F} \cong D_{X}
$$

Let $X$ and $Y$ be indecomposable $A^{F}$-modules. Up to isomorphism, we may assume that $X=\tilde{M}^{F}$ and $Y=\tilde{N}^{F}$ for some indecomposable $A$-modules $M$ and $N$ (see the proof of Theorem [5.1). In fact, we may choose $\tilde{M}=X_{k}$ and $\tilde{N}=Y_{k}$ so that $M$ and $N$ are indecomposable direct summands of the $A$-modules $X_{k}$ and $Y_{k}$, respectively. We then have

$$
D_{X} \cong\left(D_{\tilde{M}}\right)^{F} \cong \mathbb{F}_{q^{r_{1}}} \text { and } D_{Y} \cong\left(D_{\tilde{N}}\right)^{F} \cong \mathbb{F}_{q^{r_{2}}}
$$

where $r_{1}=p(M)$ and $r_{2}=p(N)$. Moreover, the Frobenius map $F_{(\tilde{M}, \tilde{N})}$ on $\operatorname{Hom}_{A}(\tilde{M}, \tilde{N})$ induces Frobenius maps on $\operatorname{Rad}_{A}^{n}(\tilde{M}, \tilde{N})$ for each $n \geqslant 1$, and thus, a Frobenius map $F$ on $\operatorname{Irr}_{A}(\tilde{M}, \tilde{N})$. By viewing $\left(\operatorname{Irr}_{A}(\tilde{M}, \tilde{N})\right)^{F}$ as a $D_{Y^{-}} D_{X^{-}}$ bimodule via the isomorphisms $\left(D_{\tilde{M}}\right)^{F} \cong D_{X}$ and $\left(D_{\tilde{N}}\right)^{F} \cong D_{Y}$, we have the following lemma.

Lemma 8.2. Let $X=\tilde{M}^{F}$ and $Y=\tilde{N}^{F}$ be indecomposable $A^{F}$-modules, where $M$ and $N$ are indecomposable A-modules. Then the $D_{Y}-D_{X}$-bimodules $\operatorname{Irr}_{A^{F}}(X, Y)$ and $\left(\operatorname{Irr}_{A}(\tilde{M}, \tilde{N})\right)^{F}$ are isomorphic.

Proof. It is clear to see that the restriction of the linear isomorphism given in Proposition 3.4(b) gives an $\mathbb{F}_{q}$-linear isomorphism

$$
\Psi: \operatorname{Rad}_{A^{F}}(X, Y) \stackrel{\sim}{\longrightarrow}\left(\operatorname{Rad}_{A}(\tilde{M}, \tilde{N})\right)^{F} ; f \longmapsto f \otimes 1,
$$

and hence, an $\mathbb{F}_{q}$-linear injection

$$
\Psi: \operatorname{Rad}_{A^{F}}^{2}(X, Y) \rightarrow\left(\operatorname{Rad}_{A}^{2}(\tilde{M}, \tilde{N})\right)^{F} .
$$

Thus, $\Psi$ induces a surjective map

$$
\bar{\Psi}: \operatorname{Irr}_{A^{F}}(X, Y) \rightarrow\left(\operatorname{Rad}_{A}(\tilde{M}, \tilde{N})\right)^{F} /\left(\operatorname{Rad}_{A}^{2}(\tilde{M}, \tilde{N})\right)^{F} \cong\left(\operatorname{Irr}_{A}(\tilde{M}, \tilde{N})\right)^{F},
$$

which is clearly a $D_{Y}-D_{X}$-bimodule homomorphism. We now prove that $\bar{\Psi}$ is a linear isomorphism by a comparison of dimensions. 
For each $0 \leqslant s \leqslant p(M)-1$ and each $0 \leqslant t \leqslant p(N)-1$, we set $n_{s t}=$ $\operatorname{dim}_{k} \operatorname{Irr}_{A}\left(M^{[s]}, N^{[t]}\right)$. Since

$$
\operatorname{Irr}_{A}(\tilde{M}, \tilde{N}) \cong \bigoplus_{\substack{0 \leqslant s \leqslant p(M)-1 \\ 0 \leqslant \leqslant \leqslant(N)-1}} \operatorname{Irr}_{A}\left(M^{[s]}, N^{[t]}\right)
$$

we have $n:=\operatorname{dim}_{k} \operatorname{Irr}_{A}(\tilde{M}, \tilde{N})=\sum_{s, t} n_{s t}$, and so $\operatorname{dim}_{\mathbb{F}_{q}}\left(\operatorname{Irr}_{A}(\tilde{M}, \tilde{N})\right)^{F}=n$. Now, take a minimal right almost split map $L \rightarrow N$. Then, so is $L^{[t]} \rightarrow N^{[t]}$ by Lemma 7.3. For each fixed $s$, it holds that $L^{[t]} \cong n_{s t} M^{[s]} \oplus L_{s, t}$ with $M^{[s]} \nmid L_{s, t}$ for all $0 \leqslant t \leqslant p(N)-1$. Thus, $\tilde{L} \cong n_{s} M^{[s]} \oplus L_{s}$ for all $s$ with $0 \leqslant s \leqslant p(M)-1$, where $n_{s}=\sum_{t=0}^{p(N)-1} n_{s t}$ and $L_{s}=\bigoplus_{t=0}^{p(N)-1} L_{s, t}$. Since $\tilde{L}=\tilde{L}^{[1]} \cong n_{s} M^{[s+1]} \oplus L_{s}^{[1]}$ and the $M^{[s]}$ are pairwise non-isomorphic, it follows that $n_{s}=n_{s+1}$ for all $0 \leqslant s<p(M)-1$. Thus, $n_{s}=\frac{n}{p(M)}$ and $\tilde{L} \cong \frac{n}{p(M)} \tilde{M} \oplus \tilde{L}^{\prime}$ for some $F$-stable module $\tilde{L}^{\prime}$ with $\tilde{M} \nmid \tilde{L}^{\prime}$.

On the other hand, by Theorem 7.4 (see also Remarks 8.1(2)), $\tilde{L}^{F} \rightarrow \tilde{N}^{F}=Y$ is minimal right almost split and

$$
\tilde{L}^{F} \cong \frac{n}{p(M)} \tilde{M}^{F} \oplus \tilde{L}^{\prime F}=\frac{n}{p(M)} X \oplus \tilde{L}^{\prime F}
$$

with $X \nmid \tilde{L}^{\prime F}$, it follows that if $\left(d, d^{\prime}\right)$ is the valuation of the arrow $[X] \rightarrow[Y]$ in the AR-quiver $\mathcal{Q}_{A^{F}}$ of $A^{F}$, then $d^{\prime}=\frac{n}{p(M)}$. Hence,

$$
\operatorname{dim}_{\mathbb{F}_{q}} \operatorname{Irr}_{A^{F}}(X, Y)=d^{\prime} \operatorname{dim}_{\mathbb{F}_{q}} D_{X}=n=\operatorname{dim}_{\mathbb{F}_{q}}\left(\operatorname{Irr}_{A}(\tilde{M}, \tilde{N})\right)^{F} .
$$

Consequently, $\bar{\Psi}$ is a $D_{Y}$ - $D_{X}$-bimodule isomorphism.

Since the algebra $A$ is defined over the algebraically close field $k=\overline{\mathbb{F}}_{q}$, we may regard the AR-quiver $\mathcal{Q}=\mathcal{Q}_{A}$ of $A$ as an ordinary quiver (see Remark 8.1(3)). We first observe that $\mathcal{Q}$ admits an admissible automorphism $\mathfrak{s}$. For each vertex $[M] \in \mathcal{Q}, \mathfrak{s}([M])$ is defined to be $\left[M^{[1]}\right]$. If $M$ and $N$ are indecomposable $A$-modules, then there are $n_{s t}$ arrows $\gamma_{s, t}^{(m)}$ from $\left[M^{[s]}\right]$ to $\left[N^{[t]}\right]$ in $\mathcal{Q}$, where $0 \leqslant s \leqslant p(M)-1$, $0 \leqslant t \leqslant p(N)-1, n_{s t}=\operatorname{dim}_{k} \operatorname{Irr}_{A}\left(M^{[s]}, N^{[t]}\right)$ and $1 \leqslant m \leqslant n_{s t}$. Note that $n_{s t}=n_{s+1, t+1}$ for all $s, t$, where subscripts are considered as integers modulo $p(M)$ and $p(N)$, respectively. We now define

$$
\mathfrak{s}\left(\gamma_{s, t}^{(m)}\right)=\gamma_{s+1, t+1}^{(m)} \text { for all } 0 \leqslant s \leqslant p(M)-1 \text { and } 0 \leqslant t \leqslant p(N)-1 .
$$

Clearly, $\mathfrak{s}$ is an admissible quiver automorphism and $(\mathcal{Q}, \mathfrak{s})$ is an ad-quiver.

Associated to $(\mathcal{Q}, \mathfrak{s})$, we may define a modulated quiver $\mathfrak{M}_{\mathcal{Q}, \mathfrak{s}}$ as in (6.2.3): let $\mathcal{A}=k \mathcal{Q}$ denote the path algebra of $\mathcal{Q}$ and $F=F_{\mathcal{Q}, \mathfrak{s}}$ be the Frobenius morphism of $\mathcal{A}$ induced by the automorphism $\mathfrak{s}$. For each vertex $\mathbf{i}(M)$ (i.e., the $\mathfrak{s}$-orbit of $[M]$ ) and each arrow $\boldsymbol{\rho}$ (i.e., an $\mathfrak{s}$-orbit of arrows in $\mathcal{Q}$ ) in $\Gamma(\mathcal{Q}, \mathfrak{s})$, we define subspaces

$$
\mathcal{A}_{\mathbf{i}(M)}=\bigoplus_{s=0}^{p(M)-1} k e_{\left[M^{[s]}\right]} \text { and } \mathcal{A}_{\boldsymbol{\rho}}=\bigoplus_{\rho \in \boldsymbol{\rho}} k \rho,
$$

of $\mathcal{A}$, which are obviously $F$-stable. By definition, the $\mathbb{F}_{q}$-modulation $\mathbb{M}(\mathcal{Q}, \mathfrak{s})$ is given by $\left(\mathcal{A}_{\mathbf{i}(M)}\right)^{F}$ and $\left(\mathcal{A}_{\boldsymbol{\rho}}\right)^{F}$ for all vertices $\mathbf{i}(M)$ and arrows $\boldsymbol{\rho}$ in $\Gamma(\mathcal{Q}, \mathfrak{s})$.

Recall Definition 6.2 of isomorphisms for modulated quivers. We can now state the following result. 
Theorem 8.3. The modulated quiver $\mathfrak{M}_{\mathcal{Q}, \mathfrak{s}}$ associated to the $A R$-quiver $(\mathcal{Q}, \mathfrak{s})$ of $A$ defined above is isomorphic to the AR-quiver $\mathcal{Q}_{A^{F}}$ of $A^{F}$. Moreover, the AuslanderReiten translation of $A$ naturally induces that of the fixed point algebra $A^{F}$.

Proof. If $X=\tilde{M}^{F}$ is an indecomposable $A^{F}$-module, then clearly, the correspondence $[X] \longrightarrow \mathbf{i}(M)$ gives a bijection between vertices of $\mathcal{Q}_{A^{F}}$ and those of $\mathfrak{M}_{\mathcal{Q}, \mathfrak{s}}$ (see Theorem 5.1). Moreover, there is an isomorphism

$$
\mathcal{A}_{\mathbf{i}(M)}=\bigoplus_{s=0}^{p(M)-1} k e_{\left[M^{[s]}\right]} \longrightarrow \bigoplus_{s=0}^{p(M)-1} D_{M^{[s]}} \cong D_{\tilde{M}}, \sum_{s} x_{s} e_{\left[M^{[s]}\right]} \longmapsto\left(x_{s} 1_{M^{[s]}}\right)_{s}
$$

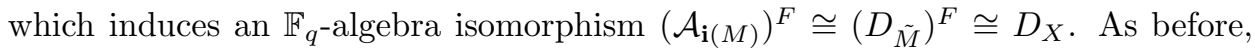
we shall identify $\left(\mathcal{A}_{\mathbf{i}(M)}\right)^{F}$ with $D_{X}$. Thus, $\left(\mathcal{A}_{\boldsymbol{\rho}}\right)^{F}$ is a $D_{Y^{-}}-D_{X}$-bimodule.

It remains to prove that for $X=\tilde{M}^{F}$ and $Y=\tilde{N}^{F}$, where $M$ and $N$ are indecomposable $A$-modules, there is a $D_{Y}$ - $D_{X}$-bimodule isomorphism

$$
\bigoplus_{\rho: \mathbf{i}(M) \rightarrow \mathbf{i}(N)}\left(\mathcal{A}_{\rho}\right)^{F} \cong \operatorname{Irr}_{A^{F}}(X, Y) .
$$

By Lemma 8.2, it suffices to show that we have a $\left(D_{\tilde{N}}\right)^{F}-\left(D_{\tilde{M}}\right)^{F}$-bimodule isomorphism

$$
\bigoplus_{\rho: \mathbf{i}(M) \rightarrow \mathbf{i}(N)}\left(\mathcal{A}_{\boldsymbol{\rho}}\right)^{F} \cong\left(\operatorname{Irr}_{A}(\tilde{M}, \tilde{N})\right)^{F}
$$

or a $D_{\tilde{N}}-D_{\tilde{M}}$-bimodule isomorphism

$$
\phi: \bigoplus_{\rho: \mathbf{i}(M) \rightarrow \mathbf{i}(N)} \mathcal{A}_{\rho} \stackrel{\sim}{\longrightarrow} \operatorname{Irr}_{A}(\tilde{M}, \tilde{N}),
$$

which is compatible with the Frobenius morphism $F_{\mathcal{A}}$ on $\mathcal{A}$ and $F$ on $\operatorname{Irr}_{A}(\tilde{M}, \tilde{N})$.

Let $d$ and $l$ denote the greatest common divisor and the least common multiple of $r_{1}=p(M)$ and $r_{2}=p(N)$, respectively. As before, let $n_{s t}=\operatorname{dim}_{k} \operatorname{Irr}_{A}\left(M^{[s]}, N^{[t]}\right)$ and denote by $\gamma_{s, t}^{(m)}$ all arrows from $\left[M^{[s]}\right]$ to $\left[N^{[t]}\right]$ in $\mathcal{Q}_{A}$. Then the set of arrows

$$
\left\{\gamma_{s, t}^{(m)} \mid 0 \leqslant s \leqslant r_{1}-1,0 \leqslant t \leqslant r_{2}-1,1 \leqslant m \leqslant n_{s t}\right\}
$$

is $\mathfrak{s}$-stable, and the arrows $\gamma_{0, t}^{(m)}$ with $0 \leqslant t \leqslant d-1$ and $1 \leqslant m \leqslant n_{0 t}$ form a complete set of representatives of $\mathfrak{s}$-orbits in this set, each of which is of length $l$. On the other hand, for each $0 \leqslant t \leqslant d-1$, the $\operatorname{space}_{\operatorname{Irr}_{A}}\left(M, N^{[t]}\right)$ is $F^{l}$-stable with $l$ minimal. Thus, we can choose a $k$-basis $\xi_{0, t}^{(m)}, 1 \leqslant m \leqslant n_{0 t}$, of $\operatorname{Irr}_{A}\left(M, N^{[t]}\right)$ which are $F^{l}$-fixed. Then the set

$$
\left\{F^{a}\left(\xi_{0, t}^{(m)}\right) \mid 0 \leqslant t \leqslant d-1,1 \leqslant m \leqslant n_{0 t}, 0 \leqslant a \leqslant l-1\right\}
$$

is a $k$-basis for $\bigoplus_{s, t} \operatorname{Irr}_{A}\left(M^{[s]}, N^{[t]}\right) \cong \operatorname{Irr}_{A}(\tilde{M}, \tilde{N})$. Thus, the correspondence $\gamma_{0, t}^{(m)} \longmapsto \xi_{0, t}^{(m)}$ induces an isomorphism of $k$-spaces

$$
\phi: \bigoplus_{\rho: \mathbf{i}(M) \rightarrow \mathbf{i}(N)} \mathcal{A}_{\boldsymbol{\rho}}=\bigoplus_{s, t, m} k \gamma_{s, t}^{(m)} \stackrel{\sim}{\longrightarrow} \operatorname{Irr}_{A}(\tilde{M}, \tilde{N})
$$

sending $\gamma_{s, t+s}^{(m)}$ to $F^{s}\left(\xi_{0, t}^{(m)}\right)$. Therefore, $\phi \circ F_{\mathcal{A}}=F \circ \phi$. Using the isomorphism given in (8.3.1), we easily see that $\phi$ is a $D_{\tilde{N}}-D_{\tilde{M}}$-bimodule isomorphism. So (8.3.3) is proved. 
To prove the last assertion, we first note that the Frobenius morphism $F_{A}$ on $A$ is also a Frobenius morphism on $A^{\mathrm{op}}$. Since the projective cover of an $A$-module is unique up to isomorphism, each $F$-stable $A$-module $M$ admits a minimal projective presentation $P_{1} \stackrel{f}{\longrightarrow} P_{0} \stackrel{\pi}{\longrightarrow} M \longrightarrow 0$ such that $P_{0}$ and $P_{1}$ are $F$-stable projective and that $f$ and $\pi$ are morphisms in $\bmod ^{F}-A$. Thus the transpose Tr $: \underline{\bmod }-A \rightarrow \underline{\bmod }-A^{\text {op }}$ induces a functor $\mathcal{T}: \underline{\bmod }^{F}-A \rightarrow \underline{\bmod }^{F}-A^{\text {op }}$, where, for example, $\underline{\bmod }^{F}-A$ denotes the factor category of $\bmod ^{F}-A$ modulo morphisms factoring through an $F$-stable projective $A$-module. It is obvious that the dual $D=\operatorname{Hom}_{k}(-, k): \bmod -A \rightarrow \bmod -A^{\mathrm{op}}$ induces a dual $\mathcal{D}: \underline{\bmod }^{F}-A \rightarrow \overline{\mathbf{m o d}}^{F}-A^{\mathrm{op}}$. On the other hand, for the fixed algebras $A^{F}$ and $\left(A^{\mathrm{op}}\right)^{F}=\left(A^{F}\right)^{\mathrm{op}}$, we also have the transpose $\operatorname{Tr}^{F}: \underline{\bmod }-A^{F} \rightarrow \underline{\bmod }-\left(A^{F}\right)^{\text {op }}$ and the dual $D^{F}=\operatorname{Hom}_{\mathbb{F}_{q}}\left(-, \mathbb{F}_{q}\right)$ : $\underline{\bmod }-A^{F} \rightarrow \overline{\bmod }-\left(A^{F}\right)^{\mathrm{op}}$. Furthermore, the functor $\Phi=\Phi_{A}: \bmod ^{F}-A \rightarrow$ mod- $A^{F}$ defined in the proof of Theorem 3.2 induces functors $\Phi_{A}: \underline{\bmod }^{F}-A \rightarrow$ $\underline{\bmod }-A^{F}$ and $\Phi_{A}: \overline{\bmod }^{F}-A \rightarrow \overline{\mathbf{m o d}}-A^{F}$. By Proposition 3.4 we finally have the following commutative square



Remarks 8.4. (1) By the definition of $\mathfrak{s}$, each $\left(\mathcal{A}_{\boldsymbol{\rho}}\right)^{F}$ is a simple $D_{Y^{-}}-D_{X}$-bimodule. Thus, the isomorphism in (8.3.2) gives a decomposition of the $D_{Y^{-}}-D_{X}$-bimodule $\operatorname{Irr}_{A^{F}}(X, Y)$ into the sum of simples.

(2) By Theorems 7.4 and 8.3, we see that the Auslander-Reiten theory for algebras defined over a finite field $\mathbb{F}_{q}$ is completely determined by the theory for algebras defined over the algebraic closure $\overline{\mathbb{F}}_{q}$.

(3) We point out that certain behaviour of Auslander-Reiten sequences under base field extension has been investigated in [27.

\section{Counting the number of $F$-Stable Representations}

In this section, we shall use the theory established in $\S 6$ to count the number of representations (resp. indecomposable representations) of a finite-dimensional hereditary (basic) $\mathbb{F}_{q}$-algebra in terms of representations of an ad-quiver. In particular, we shall prove that, for a given dimension vector, these numbers are polynomials in $q$. This generalizes [19, Corollary 3.5] to arbitrary $\mathbb{F}_{q}$-modulated quiver.

Let $(Q, \sigma)$ be an ad-quiver and let $\mathfrak{M}_{Q, \sigma}$ be the associated modulated quiver with underlying valued quiver $\Gamma=\Gamma(Q, \sigma)=\left(I, \Gamma_{1}\right)$. By definition, a representation $V=(V, \phi)$ of $Q$ over $k$ consists of a $Q_{0}$-graded $k$-vector space $V=\bigoplus_{i \in Q_{0}} V_{i}$ and a family of $k$-linear maps $\phi=\left(\phi_{\rho}\right)_{\rho}$ with $\phi_{\rho}: V_{i} \rightarrow V_{j}$ for every arrow $\rho: i \rightarrow j$ in $Q_{1}$. A morphism from $(V, \phi)$ to $\left(V^{\prime}, \phi^{\prime}\right)$ is given by a $Q_{0}$-graded morphism $f=\left(f_{i}\right)_{i}: V \rightarrow V^{\prime}$ such that $f_{t \rho} \circ \phi_{\rho}=\phi_{\rho}^{\prime} \circ f_{h \rho}$ for each arrow $\rho$. We denote by $\operatorname{Rep} Q=\operatorname{Rep}_{k} Q$ the category of all finite-dimensional representations of $Q$ over $k$. 
It is well known that $\bmod -A$, where $A=k Q$, and $\operatorname{Rep} Q$ are equivalent categories. For each $Q_{0}$-graded vector space $V=\bigoplus_{i \in Q_{0}} V_{i}$, we call

$$
\operatorname{dim} V:=\sum_{i \in I}\left(\operatorname{dim}_{k} V_{i}\right) i \in \mathbb{N} Q_{0}
$$

the dimension vector of $V$.

Following Lusztig [30], by $\mathcal{C}_{I}$ we denote the sub-category of the category $\mathcal{V}_{k, \mathbb{F}_{q}}$ (see the definition before Lemma 2.3) whose objects are $Q_{0}$-graded $k$-vector spaces $V=\bigoplus_{i \in Q_{0}} V_{i}$ with an $\mathbb{F}_{q}$-rational structure determined by a Frobenius map $F=$ $F_{V}: V \rightarrow V$ such that $F\left(V_{i}\right)=V_{\sigma(i)}$ for each $i \in Q_{0}$. The morphisms in $\mathcal{C}_{I}$ are $k$-linear maps compatible with both the gradings and the Frobenius maps. Note that, for each $\alpha=\sum_{i \in Q_{0}} a_{i} i \in \mathbb{N} Q_{0}$ satisfying $a_{i}=a_{\sigma(i)}, \forall i \in Q_{0}$, there is a unique, up to isomorphism, object in $\mathcal{C}_{I}$ with dimension vector $\alpha$ (see the remarks before Lemma 2.3.

A representation $(V, \phi)$ of $Q$ with $V \in \mathcal{C}_{I}$ is called $F$-stable if, for each $\rho \in Q_{1}$, the equality $F \circ \phi_{\rho}=\phi_{\sigma(\rho)} \circ F$ holds, that is, the following diagram commutes:

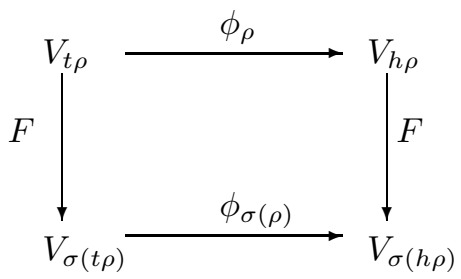

Let $\operatorname{Rep}^{F}(Q, \sigma)$ be the category consisting of $F$-stable representations of $Q$ together with morphisms in $\operatorname{Rep} Q$ which are compatible with Frobenius maps. Then $\operatorname{Rep}^{F}(Q, \sigma)$ is an abelian $\mathbb{F}_{q}$-category. It is easy to see that the equivalence between $\bmod -A$ and $\operatorname{Rep} Q$ induces an equivalence between $\bmod ^{F}$ - $A$, where $F=F_{Q, \sigma, q}$ and $\operatorname{Rep}^{F}(Q, \sigma)$. Hence, by Theorem $3.2, \operatorname{Rep}^{F}(Q, \sigma)$ and $\bmod -A^{F}$ are equivalent.

The quiver automorphism $\sigma$ extends linearly to a group automorphism $\sigma$ on $\mathbb{Z} Q_{0}$ defined by $\sigma\left(\sum_{i \in Q_{0}} a_{i} i\right)=\sum_{i \in Q_{0}} a_{i} \sigma(i)$. Clearly, if $V$ is $F$-stable, then $\sigma(\operatorname{dim} V)=\operatorname{dim} V$. Let $\left(\mathbb{Z} Q_{0}\right)^{\sigma}$ denote the $\sigma$ fixed point subset of $\mathbb{Z} Q_{0}$. This set can be identified with the group $\mathbb{Z} I$ via the canonical bijection

$$
\hat{\sigma}:\left(\mathbb{Z} Q_{0}\right)^{\sigma} \rightarrow \mathbb{Z} I ; \quad \sum_{i \in Q_{0}} b_{i} i \mapsto \sum_{\mathbf{i} \in I} a_{\mathbf{i}} \mathbf{i},
$$

where $a_{\mathbf{i}}:=b_{i}=b_{j}$ for all $i, j \in \mathbf{i}$ (see [21, §2]). In particular, the dimension vector $\operatorname{dim} X=\sum_{\mathbf{i} \in I} d_{\mathbf{i}} \mathbf{i} \in \mathbb{N} I$ of an $A^{F}$-module $X$ can be defined by

$$
\operatorname{dim} X=\hat{\sigma}(\operatorname{dim}(X \otimes k)) .
$$

Note that if, for each $\mathbf{i} \in I, S_{\mathbf{i}}$ denotes the simple $A^{F}$-module corresponding to the idempotent $e_{\mathbf{i}}=\sum_{i \in \mathbf{i}} e_{i}$ of $A^{F}$, then $d_{\mathbf{i}}=\operatorname{dim}_{\mathbb{F}_{q} \varepsilon_{\mathbf{i}}} e_{\mathbf{i}} X$ is the number of composition factors isomorphic to $S_{\mathrm{i}}$ in a composition series of $X$.

Given a matrix $x=\left(x_{i j}\right) \in k^{m \times n}$ and an integer $r \geqslant 0$, we define

$$
x^{[r]}=\left(x_{i j}^{q^{r}}\right) \in k^{m \times n} .
$$

For each $\beta=\sum_{i \in Q_{0}} b_{i} i \in\left(\mathbb{N} Q_{0}\right)^{\sigma}$, let $V_{i}=k^{b_{i}}$ for each $i \in Q_{0}$. We define a Frobenius map $F$ on the $Q_{0}$-graded vector space $V=\bigoplus_{i \in Q_{0}} V_{i}$ such that, for 
$v \in V_{i}, F(v)=v^{[1]} \in V_{\sigma(i)}$ for all $i \in Q_{0}$. We further define

$$
R(\beta)=R(Q, \beta)=\prod_{\rho \in Q_{1}} \operatorname{Hom}_{k}\left(k^{b_{t \rho}}, k^{b_{h \rho}}\right) \cong \prod_{\rho \in Q_{1}} k^{b_{h \rho} \times b_{t \rho}} .
$$

Then the Frobenius map $F$ on $V$ induces a Frobenius map on the variety $R(\beta)$ such that, for $x=\left(x_{\rho}\right) \in R(\beta), F(x)=\left(y_{\rho}\right)$ is defined by

$$
y_{\rho}(F(v))=F\left(x_{\sigma^{-1}(\rho)}(v)\right) \text { for all } \rho \in Q_{1}, v \in V_{\sigma^{-1}(t \rho)} .
$$

By viewing each $x_{\rho}$ as a matrix over $k$, we have $y_{\sigma(\rho)}=x_{\rho}^{[1]}$. Obviously, a point $x=\left(x_{\rho}\right)_{\rho}$ of $R(\beta)$ determines a representation $V(x)=\left(V_{i}, x_{\rho}\right)$ of $Q$. The algebraic subgroup

$$
G(\beta)=\prod_{i \in Q_{0}} G L_{b_{i}}(k)
$$

of $G L(V)$ acts on $R(\beta)$ by conjugation

$$
\left(g_{i}\right)_{i} \cdot\left(x_{\rho}\right)_{\rho}=\left(g_{h \rho} x_{\rho} g_{t \rho}^{-1}\right)_{\rho}
$$

and the $G(\beta)$-orbits $\mathcal{O}_{x}$ in $R(\beta)$ correspond bijectively to the isoclasses $[V(x)]$ of representations of $Q$ with dimension vector $\beta$.

The Frobenius map $F$ on $V$ also induces a Frobenius map on the group $G L(V)$ given by

$$
F(g v)=F(g)(F(v)) \text { for all } \quad g \in G L(V), v \in V .
$$

It is clear that the subgroup $G(\beta)$ is $F$-stable such that $F(g)_{\sigma(i)}=g_{i}^{[1]}$ for $g=$ $\left(g_{i}\right) \in G(\beta)$. The action of $G(\beta)$ on $R(\beta)$ restricts to an action of $G(\beta)^{F}$ on $R(\beta)^{F}$. Then, the $G(\beta)^{F}$-orbits in $R(\beta)^{F}$ correspond bijectively to the isoclasses of $F$ stable representations in $\operatorname{Rep}^{F}(Q, \sigma)$ with dimension vector $\beta$, or equivalently, to the isoclasses of $A^{F}$-modules with dimension vector $\hat{\sigma}(\beta)$.

For each $\alpha=\sum_{\mathbf{i} \in I} a_{\mathbf{i}} \mathbf{i} \in \mathbb{N} I$, let $M_{Q, \sigma}(\alpha, q)$ (resp. $I_{Q, \sigma}(\alpha, q)$ ) be the number of isoclasses of $A^{F}$-modules (resp. indecomposable $A^{F}$-modules) of dimension vector $\alpha$. Further, as indicated above, $M_{Q, \sigma}(\alpha, q)$ is the number of $G(\beta)^{F}$-orbits in $R(\beta)^{F}$, where $\beta=\hat{\sigma}^{-1}(\alpha) \in \mathbb{N} Q_{0}$.

Write $\beta=\sum_{i \in Q_{0}} b_{i} i$. Note that $b_{i}=a_{\mathbf{i}}$ for all $i \in \mathbf{i}$. For each $\mathbf{i} \in I$ and each arrow $\boldsymbol{\rho}: \mathbf{i} \rightarrow \mathbf{j}$ in $\Gamma$, we define

$$
G_{\mathbf{i}}=\prod_{i \in \mathbf{i}} G L_{b_{i}}(k) \text { and } R_{\boldsymbol{\rho}}=\prod_{\rho \in \boldsymbol{\rho}} \operatorname{Hom}_{k}\left(k^{b_{t \rho}}, k^{b_{h \rho}}\right) \cong \prod_{\rho \in \boldsymbol{\rho}} k^{b_{h \rho} \times b_{t \rho}} .
$$

By fixing an $i_{0} \in \mathbf{i}$ and a $\rho_{0} \in \boldsymbol{\rho}$, we can identify

$$
G_{\mathbf{i}}^{F}=\left\{\left(g_{i}\right)_{i \in \mathbf{i}} \mid g_{\sigma(i)}=g_{i}^{[1]} \text { for all } i \in \mathbf{i}\right\}
$$

with $G L_{a_{\mathbf{i}}}\left(\mathbb{F}_{q^{\varepsilon_{\mathbf{i}}}}\right)$ by $g_{\mathbf{i}}:=\left(g_{i}\right)_{i \in \mathbf{i}} \longmapsto g_{i_{0}}$ and identify

$$
R_{\boldsymbol{\rho}}^{F}=\left\{\left(x_{\rho}\right)_{\rho \in \boldsymbol{\rho}} \mid x_{\sigma(\rho)}=x_{\rho}^{[1]} \text { for all } \rho \in \boldsymbol{\rho}\right\}
$$

with $\mathbb{F}_{q^{\varepsilon} \boldsymbol{\rho}}^{a_{\mathbf{j}} \times a_{\mathrm{i}}}$ by $x_{\boldsymbol{\rho}}:=\left(x_{\rho}\right)_{\rho \in \boldsymbol{\rho}} \longmapsto x_{\rho_{0}}$. Hence, we may identify their products

$$
G(\beta)^{F}=\prod_{\mathbf{i} \in I} G_{\mathbf{i}}^{F} \text { and } R(\beta)^{F}=\prod_{\boldsymbol{\rho} \in \Gamma_{1}} R_{\boldsymbol{\rho}}^{F}
$$

with

$$
G:=\prod_{\mathbf{i} \in I} G L_{a_{\mathbf{i}}}\left(\mathbb{F}_{q^{\varepsilon_{\mathbf{i}}}}\right) \text { and } X:=\prod_{\boldsymbol{\rho} \in \Gamma_{1}} \mathbb{F}_{q^{\varepsilon \rho}}^{a_{\mathbf{j}} \times a_{\mathbf{i}}}
$$


respectively. Under this identification, the action of $G(\beta)^{F}$ on $R(\beta)^{F}$ becomes the action of $G$ on $X$ : for $g=\left(g_{\mathbf{i}}\right) \in G$ and $x=\left(x_{\boldsymbol{\rho}}\right) \in X$,

$$
(g \cdot x)_{\boldsymbol{\rho}}=g_{\mathbf{j}}^{\left[s_{\rho}\right]} x_{\boldsymbol{\rho}}\left(g_{\mathbf{i}}^{\left[r_{\rho}\right]}\right)^{-1} \text { for each arrow } \boldsymbol{\rho}: \mathbf{i} \rightarrow \mathbf{j},
$$

where $0 \leqslant r_{\boldsymbol{\rho}} \leqslant \varepsilon_{\mathbf{i}}-1$ and $0 \leqslant s_{\boldsymbol{\rho}} \leqslant \varepsilon_{\mathbf{j}}-1$ are determined by $\rho_{0}: \sigma^{r_{\boldsymbol{\rho}}}\left(i_{0}\right) \rightarrow \sigma^{s_{\rho}}\left(j_{0}\right)$ (see Remark 6.7(1)). Then $M_{Q, \sigma}(\alpha, q)$ is the number of $G$-orbits in $X$.

For each $g=\left(g_{\mathbf{i}}\right) \in G$, we set $X^{g}=\{x \in X \mid g \cdot x=x\}$ and $G_{g}=\{h \in G \mid h g=$ $g h\}$. By Burnside's formula, we have

$$
M_{Q, \sigma}(\alpha, q)=\frac{1}{|G|} \sum_{g \in G}\left|X^{g}\right|=\sum_{g \in \operatorname{ccl}(G)} \frac{\left|X^{g}\right|}{\left|G_{g}\right|},
$$

where $\operatorname{ccl}(G)$ is a set of representatives of conjugacy classes of $G$. Further, we have

$$
\left|X^{g}\right|=\prod_{\boldsymbol{\rho}: \mathbf{i} \rightarrow \mathbf{j}}\left|X_{\boldsymbol{\rho}}^{g}\right| \text { and }\left|G_{g}\right|=\prod_{\mathbf{i} \in I}\left|G L_{a_{\mathbf{i}}}\left(\mathbb{F}_{q^{\varepsilon_{\mathbf{i}}}}\right)_{g_{\mathbf{i}}}\right|,
$$

where $X_{\boldsymbol{\rho}}^{g}=\left\{x_{\boldsymbol{\rho}} \in \mathbb{F}_{q^{\varepsilon} \boldsymbol{\rho}}^{a_{\mathbf{j}} \times a_{\mathbf{i}}} \mid g_{\mathbf{j}}^{\left[s_{\rho}\right]} x_{\boldsymbol{\rho}}=x_{\boldsymbol{\rho}} g_{\mathbf{i}}^{\left[r_{\boldsymbol{\rho}}\right]}\right\}$ and $G L_{a_{\mathbf{i}}}\left(\mathbb{F}_{q^{\varepsilon_{\mathbf{i}}}}\right)_{g_{\mathbf{i}}}=\left\{h_{\mathbf{i}} \in G L_{a_{\mathbf{i}}}\left(\mathbb{F}_{q^{\varepsilon_{\mathbf{i}}}}\right) \mid\right.$ $\left.h_{\mathbf{i}} g_{\mathbf{i}}=g_{\mathbf{i}} h_{\mathbf{i}}\right\}$.

In order to compute $M_{Q, \sigma}(\alpha, q)$, we need to deal with the conjugacy classes in $G L_{m}\left(\mathbb{F}_{q^{r}}\right)$ for $m, r \geqslant 1$ (see, for example, [28, Chap. IV]).

We denote by $\Phi\left(q^{r}\right)$ the set all irreducible polynomials in indeterminate $T$ over $\mathbb{F}_{q^{r}}$ with leading coefficient 1 , excluding the polynomial $T$, and by $\mathcal{P}$ the set of all partitions, i.e. finite sequences $\lambda=\left(\lambda_{1}, \lambda_{2}, \ldots\right)$ of non-negative integers with $\lambda_{1} \geqslant \lambda_{2} \geqslant \cdots$. For $\lambda, \mu \in \mathcal{P}$, we define $|\lambda|=\sum_{i} \lambda_{i}, b_{\lambda}(q)=\prod_{i \geqslant 1}(1-q)\left(1-q^{2}\right)$ $\cdots\left(1-q^{n_{i}}\right)$, and $\langle\lambda, \mu\rangle=\sum_{i, j} \min \left\{\lambda_{i}, \mu_{j}\right\}$, where $n_{i}$ is the number of $\lambda_{j}$ 's with $\lambda_{j}=i$.

It is known that the conjugacy classes in $G L_{m}\left(\mathbb{F}_{q^{r}}\right)$ are in one-to-one correspondence with the isoclasses of $\mathbb{F}_{q^{r}}[T]$-modules of dimension $m$. The latter is parametrized by the functions $\pi: \Phi\left(q^{r}\right) \rightarrow \mathcal{P}$ such that $\sum_{\varphi \in \Phi\left(q^{r}\right)} d(\varphi)|\pi(\varphi)|=m$, where $d(\varphi)$ denotes the degree of $\varphi$. Each such function $\pi$ corresponds to the module

$$
\bigoplus_{\varphi \in \Phi\left(q^{r}\right)} \bigoplus_{i \geqslant 1} \mathbb{F}_{q^{r}}[T] /\left(\varphi^{\pi_{i}(\varphi)}\right)
$$

where $\pi(\varphi)=\left(\pi_{1}(\varphi), \pi_{2}(\varphi), \ldots\right)$.

Let $g \in G L_{m}\left(\mathbb{F}_{q^{r}}\right)$ be in the conjugacy class corresponding to the partition function $\pi: \Phi\left(q^{r}\right) \rightarrow \mathcal{P}$. Then we have (see [28, p. 272])

$\left|G L_{m}\left(\mathbb{F}_{q^{r}}\right)_{g}\right|=\left|\left\{h \in G L_{m}\left(\mathbb{F}_{q^{r}}\right) \mid h g=g h\right\}\right|=\prod_{\varphi \in \Phi\left(q^{r}\right)} q^{r d(\varphi)\langle\pi(\varphi), \pi(\varphi)\rangle} b_{\pi(\varphi)}\left(q^{-r d(\varphi)}\right)$.

Further, let $s, t, n_{1}, n_{2}, n \geqslant 1$, be such that $n_{1} \mid n$ and $n_{2} \mid n$. Take $g_{1} \in G L_{s}\left(\mathbb{F}_{q^{n_{1}}}\right)$ and $g_{2} \in G L_{t}\left(\mathbb{F}_{q^{n_{2}}}\right)$ such that their conjugacy classes correspond respectively to partition functions $\pi^{1}: \Phi\left(q^{n_{1}}\right) \rightarrow \mathcal{P}$ and $\pi^{2}: \Phi\left(q^{n_{2}}\right) \rightarrow \mathcal{P}$. By [20, p. 253], we have



where $d(\varphi, \psi)$ denotes the degree of the greatest common divisor of $\varphi$ and $\psi$ over a common extension field of $\mathbb{F}_{q^{n_{1}}}$ and $\mathbb{F}_{q^{n_{2}}}$. (Note that $d(\varphi, \psi)$ is independent of the extension field.)

It follows that the conjugacy classes in $G=\prod_{\mathbf{i} \in I} G L_{a_{\mathbf{i}}}\left(\mathbb{F}_{q^{\varepsilon_{\mathbf{i}}}}\right)$ are in one-to-one correspondence to multi-partition functions $\pi=\left(\pi^{\mathbf{i}}\right)_{\mathbf{i} \in I}$ of $\pi^{\mathbf{i}}: \Phi\left(q^{\varepsilon_{\mathbf{i}}}\right) \rightarrow \mathcal{P}$ with 
$\sum_{\varphi \in \Phi\left(q^{\varepsilon_{\mathbf{i}}}\right)} d(\varphi)\left|\pi^{\mathbf{i}}(\varphi)\right|=a_{\mathbf{i}}$. The set of all such multi-partition functions is denoted by $\mathfrak{P}$. For each $\pi \in \mathfrak{P}$, choose an element $g^{\pi}=\left(g_{\mathbf{i}}^{\pi}\right)_{\mathbf{i}} \in G$ such that its conjugacy class corresponds to $\pi$. Thus, we have

$$
\left|G_{g^{\pi}}\right|=\prod_{\mathbf{i} \in I}\left|G L_{a_{\mathbf{i}}}\left(\mathbb{F}_{q^{\varepsilon_{\mathbf{i}}}}\right)_{g_{\mathbf{i}}^{\pi}}\right|=\prod_{\mathbf{i} \in I} \prod_{\varphi \in \Phi\left(q^{\varepsilon_{\mathbf{i}}}\right)} q^{\varepsilon_{\mathbf{i}} d(\varphi)\left\langle\pi^{\mathbf{i}}(\varphi), \pi^{\mathbf{i}}(\varphi)\right\rangle} b_{\pi^{\mathbf{i}}(\varphi)}\left(q^{-\varepsilon_{\mathbf{i}} d(\varphi)}\right) .
$$

Clearly, for each $\mathbf{i} \in I, \pi^{\mathbf{i}}$ corresponds to the conjugacy class of $g_{\mathbf{i}}^{\pi}$ in $G L_{a_{\mathbf{i}}}\left(\mathbb{F}_{q^{\varepsilon_{\mathbf{i}}}}\right)$. Let $0 \leqslant s \leqslant \varepsilon_{\mathbf{i}}-1$ and denote by $\pi^{\mathbf{i}}[s]$ the partition function $\Phi\left(q^{\varepsilon_{\mathbf{i}}}\right) \rightarrow \mathcal{P}$ corresponding to the conjugacy class of $\left(g_{\mathbf{i}}^{\pi}\right)^{[s]}$. Then we get

$$
\left|X^{g^{\pi}}\right|=\prod_{\boldsymbol{\rho}: \mathbf{i} \rightarrow \mathbf{j}}\left|X_{\boldsymbol{\rho}}^{g^{\pi}}\right|=\prod_{\boldsymbol{\rho}: \mathbf{i} \rightarrow \mathbf{j}} q^{\varepsilon_{\boldsymbol{\rho}} \sum_{\varphi \in \Phi\left(q^{\left.\varepsilon_{\mathbf{i}}\right)}, \psi \in \Phi\left(q^{\varepsilon_{\mathbf{j}}}\right)\right.} d(\varphi, \psi)\left\langle\pi^{\mathrm{i}}\left[r_{\boldsymbol{\rho}}\right](\varphi), \pi^{\mathrm{j}}\left[s_{\boldsymbol{\rho}}\right](\psi)\right\rangle}
$$

Finally, we deduce

$$
\begin{aligned}
M_{Q, \sigma}(\alpha, q) & =\sum_{\pi \in \mathfrak{P}} \frac{\left|X^{g^{\pi}}\right|}{\left|G_{g^{\pi}}\right|} \\
& =\sum_{\pi \in \mathfrak{P}} \frac{\prod_{\rho: \mathbf{i} \rightarrow \mathbf{j}} q^{\varepsilon_{\rho} \sum_{\varphi \in \Phi\left(q^{\varepsilon_{\mathbf{i}}}\right), \psi \in \Phi\left(q^{\varepsilon_{\mathbf{j}}}\right)} d(\varphi, \psi)\left\langle\pi^{\mathbf{i}}\left[r_{\rho}\right](\varphi), \pi^{\mathbf{j}}\left[s_{\rho}\right](\psi)\right\rangle}}{\prod_{\mathbf{i} \in I} \prod_{\varphi \in \Phi\left(q^{\varepsilon_{\mathbf{i}}}\right)} q^{\varepsilon_{\mathbf{i}} d(\varphi)\left\langle\pi^{\mathbf{i}}(\varphi), \pi^{\mathbf{i}}(\varphi)\right\rangle} b_{\pi^{\mathbf{i}}(\varphi)}\left(q^{-\varepsilon_{\mathbf{i}} d(\varphi)}\right)} .
\end{aligned}
$$

An orientation of the underlying graph $\bar{Q}$ of $Q$ is called $\sigma$-admissible 3 if it is compatible with the graph automorphism of $\bar{Q}$ induced by $\sigma$. Obviously, the orientation of $Q$ itself is $\sigma$-admissible.

Theorem 9.1. The number $M_{Q, \sigma}(\alpha, q)$ is a polynomial in $q$ with rational coefficients and independent of the $\sigma$-admissible orientation of $Q$.

Proof. By (9.0.2), $M_{Q, \sigma}(\alpha, q)$ is a rational function in $q$ over $\mathbb{Q}$. Since $M_{Q, \sigma}(\alpha, q) \in$ $\mathbb{Z}$ for all power $q=p^{s}$ of a prime $p$, it follows that $M_{Q, \sigma}(\alpha, q)$ is a polynomial. Further, whenever $i_{0} \in \mathbf{i}$ and $\rho_{0} \in \boldsymbol{\rho}$ for $\mathbf{i} \in I$ and $\boldsymbol{\rho} \in \Gamma_{1}$ are fixed, and the numbers $r_{\rho}$ and $s_{\rho}$ for each $\rho \in \Gamma_{1}$ are clearly independent of the orientation of $\boldsymbol{\rho}$, we have again by (9.0.2) that $M_{Q, \sigma}(\alpha, q)$ is independent of the orientation of $\Gamma$, i.e., the $\sigma$-admissible orientation of $Q$.

By induction on the height ht $\alpha:=\sum_{\mathbf{i} \in I} a_{\mathbf{i}}$ of $\alpha=\sum_{\mathbf{i} \in I} a_{\mathbf{i}} \mathbf{i} \in \mathbb{N} I$, we deduce the following; see [19] for the case of natural $\mathbb{F}_{q}$-modulated quivers (Remarks 6.7(2)).

Corollary 9.2. The number $I_{Q, \sigma}(\alpha, q)$ of isoclasses of indecomposable $A^{F}$-modules of dimension vector $\alpha$ is a polynomial in $q$ with rational coefficients and independent of the $\sigma$-admissible orientation of $Q$.

\section{KAC'S THEOREM FOR THE SYMMETRIZABLE CASE}

In this last section, we present an application to Lie theory. We keep the notation introduced in $\S 9$. Thus, $(Q, \sigma)$ denotes an ad-quiver and $\mathfrak{M}_{Q, \sigma}$ is the associated modulated quiver with the underlying valued quiver $\Gamma=\Gamma(Q, \sigma)=\left(I, \Gamma_{1}\right)$.

\footnotetext{
${ }^{3}$ The $\sigma$-admissible orientations of the underlying graph of $Q$ correspond to the orientations of the underlying graph of $\Gamma$.
} 
The quiver $Q$ defines a symmetric generalized Cartan matrix $C_{Q}=\left(a_{i j}\right)_{i, j \in Q_{0}}$ given by

$$
a_{i j}= \begin{cases}2 & \text { if } i=j, \\ -\mid\{\text { arrows between } i \text { and } j\} \mid & \text { if } i \neq j,\end{cases}
$$

while the valued quiver $\Gamma$ defines a symmetrizable generalized Cartan matrix $C_{\Gamma}=$ $\left(b_{\mathbf{i} \mathbf{j}}\right)_{\mathbf{i}, \mathbf{j} \in I}$ given by

$$
b_{\mathbf{i j}}= \begin{cases}2 & \text { if } \mathbf{i}=\mathbf{j}, \\ -\sum_{\boldsymbol{\rho}} \varepsilon_{\boldsymbol{\rho}} / \varepsilon_{\mathbf{i}} & \text { if } \mathbf{i} \neq \mathbf{j},\end{cases}
$$

where the sum is taken over all arrows $\boldsymbol{\rho}$ between $\mathbf{i}$ and $\mathbf{j}$ (see (6.2.1)). In fact, all symmetrizable generalized Cartan matrices can be obtained in this way.

Let $\Delta(\Gamma) \subset \mathbb{Z} I$ be the root system associated with the valued quiver $\Gamma$, or equivalently, the root system of the Kac-Moody algebra associated with the Cartan matrix $C_{\Gamma}$ (see 24] or 26] for its definition). We shall write $\Delta(Q)$ for $\Delta(\Gamma)$ if $\sigma=1$ and $\Delta(\Gamma)^{+}$for the positive root system of $\Delta(\Gamma)$. Putting $I_{Q}(\alpha, q)=I_{Q, 1}(\alpha, q)$ (see 9.2), it is proved in 24] that the polynomial $I_{Q}(\alpha, q)$ is non-zero if and only if $\alpha \in \Delta(Q)^{+}$. Moreover, $I_{Q}(\alpha, q)=1$ if $\alpha$ is real. This is known as Kac's theorem which links the representations of a quiver with its associated (symmetric) KacMoody algebra. We shall see below that this relation remains true for a modulated quiver and its associated Kac-Moody algebra. Thus, Kac's theorem holds in the symmetrizable case. See Remarks 10.4 below for other proofs of this generalization.

For $\beta \in \Delta(Q)$, let $t \geqslant 1$ be the minimal integer satisfying $\sigma^{t}(\beta)=\beta$. We call $t$ the $\sigma$-period of $\beta$, denoted $p(\beta)=p_{\sigma}(\beta)$. Recall from (9.0.1) the group isomorphism $\hat{\sigma}:\left(\mathbb{Z} Q_{0}\right)^{\sigma} \rightarrow \mathbb{Z} I$ which induces a map from $\Delta(Q)$ to $\Delta(\Gamma)$ in the following.

Lemma 10.1 ([34, 21, Prop. 4]). Let $\beta \in \Delta(Q)$ and let

$$
\tilde{\beta}:=\beta+\sigma(\beta)+\cdots+\sigma^{t-1}(\beta) \in\left(\mathbb{Z} Q_{0}\right)^{\sigma},
$$

where $t=p_{\sigma}(\beta)$. Then $\beta \longmapsto \hat{\sigma}(\tilde{\beta})$ defines a surjective map $\Delta(Q) \rightarrow \Delta(\Gamma)$. Moreover, if $\hat{\sigma}(\tilde{\beta})$ is real, then $\beta$ is real and is unique up to $\sigma$-orbit.

Let $A$ be the path algebra $k Q$ of $Q$ over $k=\overline{\mathbb{F}}_{q}$ and let $F=F_{Q, \sigma, q}$ be the Frobenius morphism on $A$ induced from $\sigma$. In order to prove the following result, we recall from [21, §3] the definition of an isomorphically invariant representation of $Q$. Given a representation $V=(V, \phi)$ of $Q$ over $k$, we define a new representation ${ }^{\sigma} V=\left({ }^{\sigma} V, \psi\right)$ of $Q$ by $\left({ }^{\sigma} V\right)_{i}=V_{\sigma^{-1}(i)}$ and $\psi_{\rho}=\phi_{\sigma^{-1}(\rho)}$ for all $i \in Q_{0}$ and $\rho \in Q_{1}$. The representation $V$ is called isomorphically invariant if $V \cong \sigma V$. Such a representation $V$ is said to be isomorphically invariant indecomposable if it is not a direct sum of two non-zero isomorphically invariant representations. Since the Frobenius morphism $F_{Q, \sigma, q}$ on $A$ is the composition of the standard Frobenius morphism $F_{Q, 1, q}$ and the algebra automorphism of $k Q$ induced from $\sigma$, we have the following relation between isomorphically invariant representations and $F$-stable representations.

Lemma 10.2. Let $V=(V, \phi)$ be a representation of $Q$ defined over $\mathbb{F}_{q}$ (i.e., all linear maps $\phi_{\rho}$ are defined over $\mathbb{F}_{q}$ ). Then $V$ is $F$-stable (with respect to $F_{Q, \sigma, q}$ ) if and only if $V$ is isomorphically invariant.

Proof. If $V$ is defined over $\mathbb{F}_{q}$, then the Frobenius twist $V^{\left[F_{V}\right]}$ for some Frobenius map $F_{V}$ on $V$ is isomorphic to ${ }^{\sigma} V$ (see $\S 9$ ). Now the assertion follows from Proposition 4.3(b). 
Theorem 10.3. The polynomial $I_{Q, \sigma}(\alpha, q)$ is non-zero if and only if $\alpha \in \Delta(\Gamma)^{+}$. Moreover, if $\alpha \in \Delta(\Gamma)^{+}$is real, then $I_{Q, \sigma}(\alpha, q)=1$.

Proof. Let $X$ be an indecomposable $A^{F}$-module with $\operatorname{End}_{A^{F}}(X) / \operatorname{Rad}\left(\operatorname{End}_{A^{F}}(X)\right)$ $\cong \mathbb{F}_{q^{r}}$. By Theorem [5.1, there is an indecomposable $A$-module $M$ such that $M=$ $M^{[r]}$ and

$$
X_{k}:=X \otimes_{\mathbb{F}_{q}} k \cong M \oplus M^{[1]} \oplus \cdots \oplus M^{[r-1]} .
$$

Moreover, $M, M^{[1]}, \ldots, M^{[r-1]}$ are pairwise non-isomorphic. By Kac's theorem, the dimension vector $\beta:=\operatorname{dim} M$ lies in $\Delta(Q)^{+}$. Let $t$ be minimal such that $\sigma^{t}(\beta)=\beta$. Since $\operatorname{dim} M^{[1]}=\sigma(\operatorname{dim} M)$, we have $t \mid r$ and

$$
\operatorname{dim} X_{k}=\beta+\sigma(\beta)+\cdots+\sigma^{r-1}(\beta)=\frac{r}{t} \tilde{\beta} .
$$

If $\beta$ is an imaginary root, then $\hat{\sigma}(\tilde{\beta})$ is an imaginary root in $\Delta(\Gamma)^{+}$. We conclude that

$$
\operatorname{dim} X=\hat{\sigma}\left(\operatorname{dim} X_{k}\right)=\frac{r}{t} \hat{\sigma}(\tilde{\beta})
$$

is an imaginary root in $\Delta(\Gamma)^{+}$. If $\beta$ is real, then $r=t$. This implies that $\operatorname{dim} X=$ $\hat{\sigma}(\tilde{\beta})$ is a real root in $\Delta(\Gamma)^{+}$.

Conversely, let $\alpha \in \Delta(\Gamma)^{+}$. Then by [21, Thm 1], there is a representation $V$ of $Q$ over an algebraically closed field $k=\overline{\mathbb{F}}_{p}$ for some prime $p$ ( $p$ does not divide the order of $\sigma$ ) such that $V$ is isomorphically invariant indecomposable of dimension vector $\hat{\sigma}^{-1}(\alpha)$. Suppose $V$ and all its summands are defined over a finite extension $\mathbb{F}_{q}$ of $\mathbb{F}_{p}$, where $q=p^{s}$ for some $s \geqslant 1$. Then $V$ is $F$-stable by Lemma 10.2 with respect to the Frobenius morphism $F=F_{Q, \sigma, q}$ on $A=k Q$. This gives rise to an $A^{F}$-module $V^{F}$ of dimension vector $\alpha$. We claim that $V^{F}$ is an indecomposable. Indeed, suppose $V^{F}=X_{1} \oplus X_{2}$ with $X_{1} \neq 0 \neq X_{2}$. Then

$$
V=V^{F} \otimes_{\mathbb{F}_{q}} k \cong\left(X_{1} \otimes_{\mathbb{F}_{q}} k\right) \oplus\left(X_{2} \otimes_{\mathbb{F}_{q}} k\right) .
$$

Since both $X_{1} \otimes_{\mathbb{F}_{q}} k$ and $X_{2} \otimes_{\mathbb{F}_{q}} k$ are $F$-stable and defined over $\mathbb{F}_{q}$, they are isomorphically invariant by Lemma 10.2 again. This contradicts the fact that $V$ is isomorphically invariant indecomposable. Hence, $V^{F}$ is an indecomposable $A^{F}$ module of dimension vector $\alpha$. This implies that the polynomial $I_{Q, \sigma}(\alpha, q)$ is nonzero.

Now let $\alpha=\hat{\sigma}(\tilde{\beta})$ be a real root. Then, by Lemma 10.1, $\beta$ is real. Again by Kac's theorem, there is a unique indecomposable $A$-module $M$ with dimension vector $\beta$. Since $\operatorname{dim} M^{[t]}=\sigma^{t}(\operatorname{dim} M)=\operatorname{dim} M$, where $t$ is minimal such that $\sigma^{t}(\beta)=\beta$, we have $M^{[t]} \cong M$. Hence, by Theorem [5.1, we obtain an $F$-stable module

$$
\tilde{M} \cong M \oplus M^{[1]} \oplus \cdots \oplus M^{[t-1]}
$$

such that $\tilde{M}^{F}$ is an indecomposable $A^{F}$-module whose dimension vector is $\hat{\sigma}(\tilde{\beta})=$ $\alpha$. The uniqueness of such an indecomposable module follows from the fact that $\beta$ is unique up to $\sigma$-orbit.

Remarks 10.4. (1) In the natural $\mathbb{F}_{q}$-modulated quiver case (see Remarks 6.7), this result has been partially proved by Hua [19, Theorem 4.1] and completely proved by Hubery [21, Theorem 24]. Although Theorem 10.3 covers all $\mathbb{F}_{q}$-modulated quivers, the sufficient part in our proof is somewhat indirect, based on Theorem 1 given in [21]. 
(2) By using the Ringel-Hall algebra approach, it has been proved in 8 that, for any prime power $q$ and dimension vector $\alpha$, the number $I_{Q, \sigma}(\alpha, q) \neq 0$ if and only if $\alpha \in \Delta(\Gamma)^{+}$. This is stronger than the statement in Theorem 10.3 .

It should still be interesting to find a direct proof of Kac's theorem in the modulated quiver case. We end the paper with the following conjecture which provides such a direct link of Kac's theorem with its generalization.

Conjecture 10.5. Let $A$ be the path algebra of an ad-quiver $(Q, \sigma)$ without oriented cycles. Let $\beta \in \Delta(Q)^{+}$. Then there exists an indecomposable A-module $M$ with dimension vector $\beta$ such that $p_{F}(M)=p_{\sigma}(\beta)$.

We claim that this conjecture immediately implies that $I_{Q, \sigma}(\alpha, q) \neq 0$ for each $\alpha \in \Delta(\Gamma)^{+}$. Indeed, for any $\alpha \in \Delta(\Gamma)^{+}$, there exists a $\beta \in \Delta(Q)^{+}$such that $\alpha=\hat{\sigma}(\tilde{\beta})$. By Kac's theorem, we have $I_{Q, 1}(\beta, q) \neq 0$ for some $q$. Now the existence of such an $M$ guarantees that the associated $F$-stable module $\tilde{M}$ defined in (5.0.1) has dimension vector $\tilde{\beta}$. Thus, the indecomposable module $\tilde{M}^{F}$ has dimension vector $\alpha$. Therefore, $I_{Q, \sigma}(\alpha, q) \neq 0$.

\section{ACKNOWLEDGEMENT}

The main results of the paper were presented by the second author at the Conference for Representations of Algebraic Groups, Aarhus, June 2-6, 2003, and by both authors at the Workshop on Representation Theory, Canberra, June 30-July 4, 2003. We thank the organizers for the opportunity to attend these conferences. The second author also thanks Wilberd van der Kallen for discussions on Frobenius morphisms over infinite-dimensional vector spaces.

\section{REFERENCES}

[1] M. Auslander, I. Reiten, and S.O. Smalø, Representation Theory of Artin Algebras, Cambridge Studies in Advanced Mathematics: 36. Cambridge University Press, Cambridge, 1995. MR.1314422 (96c:16015)

[2] D. Benson, Representations and Cohomology, Vol I, Cambridge Studies in Advanced Mathematics: 30. Cambridge University Press, Cambridge, 1995. MR1110581 (92m:20005)

[3] R. W. Carter, Finite groups of Lie type, John Wiley and Sons, New York, 1985. MR0794307 (87d:20060)

[4] E. Cline, B. Parshall and L. Scott, Finite dimensional algebras and highest weight categories, J. Reine Angew. Math. 391 (1988), 85-99. MR0961165 (90d:18005)

[5] C.W. Curtis and I. Reiner, Methods of representation theory. With applications to finite groups and orders, Vol. I., Wiley-Interscience, New York, 1981. MR0632548 (82i:20001)

[6] B. Deng and J. Du, Monomial bases for quantum affine $\mathfrak{s l}_{n}$, Adv. Math. 191 (2005), 276-304. MR2103214

[7] B. Deng and J. Du, Bases of quantized enveloping algebras, Pacific J. Math. 220 (2005), 33-48.

[8] B. Deng and J. Xiao, A new approach to Kac's theorem on representations of valued quivers, Math. Z. 245 (2003), 183-199. MR.2023959 (2004k:16032)

[9] F. Digne and J. Michel, Representations of finite groups of Lie type, London Math. Soc. Student Texts: 21. Cambridge University Press, Cambridge, 1991. MR1118841 (92g:20063)

[10] V. Dlab and C.M. Ringel, On algebras of finite representation type, J. Algebra 33, (1975), 306-394. MR0357506 (50:9974)

[11] V. Dlab and C.M. Ringel, Indecomposable representations of graphs and algebras, Memoirs Amer. Math. Soc. 6 no. 173, 1976. MR0447344 (56:5657)

[12] P.W. Donovan and M.R. Freislich, The representation theory of finite graphs and associated algebras, Carleton Math. Lecture Notes 5, 1973. MR0357233 (50:9701) 
[13] Y.A. Drozd and V.V. Kirichenko, Finite-dimensional algebras, Translated from the 1980 Russian original and with an appendix by Vlastimil Dlab. Springer-Verlag, Berlin, 1994. MR.1284468 (95i:16001)

[14] P. Gabriel, Unzerlegbare Darstellungen I, Manuscripta Math. 6 (1972), 71-103. MR0332887 (48:11212)

[15] P. Gabriel, Indecomposable representations II, Istit. Naz. Atta Mat., Symp. Math. XI (1973), 81-104. MR0340377 (49:5132)

[16] P. Gabriel, Auslander-Reiten sequences and representation-finite algebras, Lecture Notes in Math.: 831, Springer-Verlag, Berlin, Heidelberg, New York (1980), 1-71. MR0607140 (82i:16030)

[17] P. Gabriel and A.V. Roiter, Representations of finite dimensional algebras, Springer-Verlag, Berlin, Heidelberg, 1997. MR,1475926 (98e:16014)

[18] J. Hua, Representations of quivers over finite fields, Ph.D. thesis, University of New South Wales, 1998

[19] J. Hua, Numbers of representations of valued quivers over finite fields, preprint, Universität



[20] J. Hua and Z. Lin, Generalized Weyl denominator formula, In: Representations and quantizations, Proceedings of the International Conf. on Representation Theory (Shanghai, 1998), 247-261, China High. Educ. Press, Beijing, 2000. MR1802176 (2002d:16016)

[21] A. Hubery, Quiver representations respecting a quiver automorphism: a generalisation of a theorem of Kac, J. London Math. Soc. (2) 69 (2004), 79-96. MR.2025328 (2004k:16033)

[22] J. C. Jantzen, Representations of algebraic groups, Academic Press, New York, 1987. MR0899071 (89c:20001)

[23] C.U. Jensen and H. Lenzing, Homological dimension and representation type of algebras under base field extension, Manuscripta Math. 39 (1982), 1-13. MR0672397 (83k:16019)

[24] V. Kac, Infinite root systems, representations of graphs and invariant theory, Invent. Math. 56 (1980), 57-92. MR0557581 (82j:16050)

[25] V. Kac, Root systems, representations of quivers and invariant theory, Lecture Notes in Mathematics: 996, Springer-Verlag, 1982, 74-108. MR0718127 (85j:14088)

[26] V. Kac, Infinite dimensional Lie algebras, Third edition, Cambridge University Press, 1990. MR.1104219 (92k:17038)

[27] S. Kasjan, Auslander-Reiten sequences under base field extension, Proc. Amer. Math. Soc. 128 (2000), 2885-2896. MR1670379 (2000m:16025)

[28] I.G. Macdonald, Symmetric functions and Hall polynomials, 2nd edition, Clarendon Press, Oxford, 1995. MR.1354144 (96h:05207)

[29] G. Lusztig, Introduction to quantum groups, Progress in Math. 110, Birkhäuser, 1993. MR1227098 (94m:17016)

[30] G. Lusztig, Canonical bases and Hall algebras, Representation theories and algebraic geometry, 365-399, Kluwer Acad. Publ., Dordrecht, 1998. MR.1653038 (2000d:17020)

[31] L.A. Nazarova, Representations of quivers of infinite type, Math. USSR Izvestija Ser. Mat. 7 (1973), 752-791. MR0338018 (49:2785)

[32] C. M. Ringel, Hall algebras and quantum groups, Invent. Math. 101 (1990), 583-592. MR.1062796 (91i:16024)

[33] L. Scott, Simulating algebraic geometry with algebra, I, Proc. Symp. Pure Math. 47 (1987), 271-281. MR0933417 (89c:20062a)

[34] T. Tanisaki, Foldings of root systems and Gabriel's theorem, Tsukuba J. Math. 4 (1980), 89-97. MR0597686 (82g:16031)

Department of Mathematics, Beijing Normal University, Beijing 100875, People's Republic of China

E-mail address: dengbm@bnu.edu.cn

School of Mathematics, University of New South Wales, Sydney 2052, Australia

E-mail address: j.du@unsw.edu.au

URL: http://www. maths. unsw.edu.au/ jied 